\title{
Scaled Gromov Four-Point Condition for Network Graph Curvature Computation
}

Edmond Jonckheere, Poonsuk Lohsoonthorn, and Fariba Ariaei

Abstract. In this paper, we extend the concept of scaled Gromov hyperbolic graph, originally developed for the thin triangle condition (TTC), to the computationally simplified, but less intuitive, four-point condition (FPC). The original motivation was that for a large but finite network graph to enjoy some of the typical properties to be expected in negatively curved Riemannian manifolds, the delta measuring the thinness of a triangle scaled by its diameter must be below a certain threshold all across the graph. Here we develop various ways of scaling the 4-point delta, and develop upper bounds for the scaled 4-point delta in various spaces. A significant theoretical advantage of the TTC over the FPC is that the latter allows for a Gromov-like characterization of Ptolemaic spaces. As a major network application, it is shown that scale-free networks tend to be scaled Gromov hyperbolic, while small-world networks are rather scaled positively curved.

(C) Taylor \& Francis Group, LLC

ISSN: $1542-7951 / 08$ 


\section{Introduction}

\section{I.I. Network Congestion Motivation}

The "scaled Gromov hyperbolic" property of networks, as originally defined in [Jonckheere et al. 08], has far-reaching implications in network analysis and design, the most important one being probably congestion. This was best demonstrated in [Narayan and Saniee 09], in which the authors used the Rocketfuel database as a test bed and verified that those networks pass the $\delta_{\text {TтC }}(\Delta) / \operatorname{diam}(\Delta)<3 / 2$ scaled hyperbolicity test. Here $\Delta$ is an arbitrary geodesic triangle embedded in the network, and TTC stands for thin triangle condition, in the sense that $\delta_{\text {ттС }}(\Delta)$ is the minimum perimeter of all triangles inscribed in $\Delta$, which has to remain "small" compared with the diameter of the triangle, hence giving $\Delta$ a "thin" external appearance. Next, Narayan and Saniee implemented a synthetic traffic model (driven by a uniformly distributed demand), and experimentally observed that the Rocketfuel networks have a maximum congestion vertex $v$, where traffic (quantified by betweenness) scales as $N^{2}$, where $N$ is the order of the network. To epitomize the crucial role played by the curvature, the authors implemented the same synthetic traffic on 2-dimensional Euclidean lattices and observed that in contrast to hyperbolic networks, in this case the congestion scales as $N^{1.5}$.

Following up on this line of work, in [Jonckheere et al. 11b, Lou et al. 11], the results of Narayan and Saniee were proved on a continuous-geometry model of network traffic. A Gromov hyperbolic network was modeled as a subset $X \subset \mathbb{H}^{n}$ of the hyperbolic space, a continuous-geometry traffic function representative of the betweenness was defined, and it was proved that the maximum of the traffic over a small subset $V \subset X$ scales as $\operatorname{vol}(X)^{2}$, that is, $N^{2}$, if we identify the number of vertices in the network with the $\operatorname{volume} \operatorname{vol}(X)$ of its Riemannian-geometric counterpart. Furthermore, the maximum congestion appears for a small subset $V$ around the center of gravity of $X$ (see [Jost 97] for the concept of center of gravity of a manifold). The same methodology applied to a Euclidean subset $X \subset \mathbb{E}^{n}$ revealed a congestion scaling as $\operatorname{vol}(X)^{1+1 / n}$, hence confirming the experimental 2-dimensional result of Narayan and Saniee.

Besides the "bad" congestion implication, hyperbolicity has a "good" implication in terms of "navigability" [Boguna et al. 10].

The Gromov hyperbolic property has implications beyond the realm of classical networks - specifically, in quantum networks, where the message is encoded in spin excitation. Uniform spin chains endowed with a metric reflecting the maximum transfer probability turn out to be Gromov hyperbolic [Jonckheere et al. 11a], as in the "scaled 4-point" criterion precisely developed in the present 
paper. But in contrast to the existence of a center of gravity as in classical networks, spin chains have a "center of antigravity" that acts as a repeller of the excitation encoding the messages. The reason for this discrepancy is subtle: A classical Gromov hyperbolic network is quasi-isometric to a tree and hence has a Cantor Gromov boundary [Jonckheere et al. 07], while a Gromov hyperbolic spin chain is not quasi-isometric to a tree and hence has its Gromov boundary reduced to a singleton [Jonckheere et al. 11a].

\subsection{Scaled Gromov Hyperbolic Networks}

As already alluded to in the previous subsection, the Gromov hyperbolic property of metric spaces can be formulated in essentially two different ways. The first and most intuitive formulation, already emphasized in Section 1.1, rephrases the wellknown fact that the sum of the internal angles of a geodesic triangle $\triangle$ drawn on a negatively curved surface is less than $\pi$, endowing the triangle with a "thin" external appearance. The Gromov $\delta_{\text {ттс }}(\triangle)$ somehow quantifies how "fat" the triangle $\triangle$ is, using the more primitive concept of distance, so that $\delta_{\text {TTC }}$ applies to arbitrary metric spaces, e.g., graphs, subject to the technical requirement that the metric space be geodesic. The $\delta_{\text {TтC }}$ could be the minimum perimeter of all inscribed triangles, the diameter of the inscribed triangle also referred to as insize, the neighborhood size $\delta_{\text {тTC }}$ such that the union of $\delta_{\text {TтC }}$-neighborhoods of two sides of $\triangle$ contains the third side, etc. A geodesic metric space is then said to be Gromov hyperbolic if the thin triangle condition (TTC) holds, that is, if there exists a bound $\bar{\delta}_{\text {TTC }}$ such that $\delta_{\text {TTC }}(\triangle)<\bar{\delta}_{\text {TTC }}$ for all geodesic triangles embedded in the space. (See [Bridson and Haefliger 99, Chapter III.H] for a survey.) All of these measures, however, involve in one way or another the sides of the triangle and as such require the metric space to be geodesic.

The second formulation precisely removes this geodesic requirement; it does not involve triangle sides, but it has the added difficulty of requiring four points, $a, b, c, d$. We construct the set of all (unordered) pairs of distinct points and partition this set into three subsets of nonintersecting pairs:

$$
\{\{a, d\},\{b, c\}\} \cup\{\{a, b\},\{c, d\}\} \cup\{\{a, c\},\{b, d\}\}
$$

The two nonintersecting pairs of points in a subset of the partition are referred to as opposite, with reference to the geometric interpretation shown in Figure 1. For each subset of the partition, we compute the sum of the distances between points in pairs, and we list the sums in decreasing order as follows:

$$
L:=d(a, d)+d(b, c) \geq M:=d(a, b)+d(c, d) \geq S:=d(a, c)+d(b, d),
$$




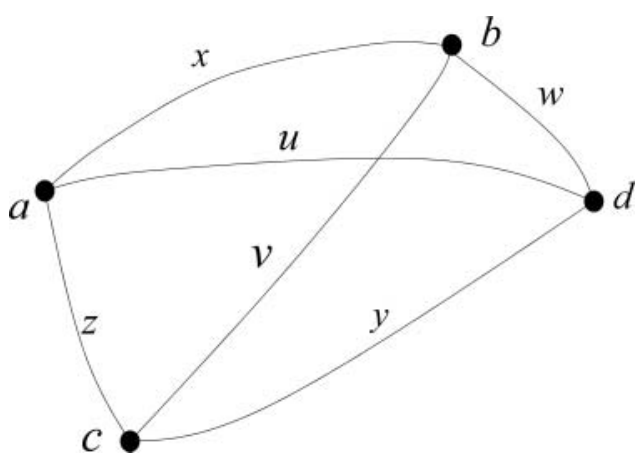

Figure I. Illustration of the various quantities when the metric space is geodesic and the distances can be interpreted as lengths of diagonals of the complete quadrilateral. For the subset $\{\{a, b\} \cup\{c, d\}\}$, the pairs of points $\{a, b\}$ and $\{c, d\}$ and the geodesic diagonals $[a, b]$ and $[c, d]$ are said to be opposite.

where $L, M, S$ are the largest, middle, and smallest such sums, respectively. We define $\delta(a, b, c, d)=(L-M) / 2$. Then an equivalent formulation of the Gromov hyperbolic property is existence of a bound $\bar{\delta}$ such that $\delta(a, b, c, d)<\bar{\delta}$ for all quadruples of points, the so-called (Gromov) four-point condition $(F P C)$.

We will sometimes refer to the quadruple $(a, b, c, d)$ as the quadrilateral $\square a b c d$, because the latter is more geometrically appealing, with a word of caution that if the space is not geodesic, there might not exist a geodesic edge $[a, b]$ of length equal to $d(a, b)$.

There are some definite computational advantages in using the FPC instead of the TTC, since the former does not require computation of geodesics (but it still requires computation of distances). However, both formulations suffer the restriction that they are not directly applicable to real-world networks, in which all graphs, no matter how awesome their sizes, have finite $\delta$ 's.

This leaves the investigator in a quandary as to how small $\delta$ should be for the graph to enjoy some Gromov hyperbolic properties. For the TTC, the directing idea was to scale $\delta_{\text {тт }}$ relative to the diameter of the triangle and declare the graph scaled Gromov hyperbolic if $\delta_{\mathrm{TTC}}(\triangle) / \operatorname{diam}(\triangle)<3 / 2, \forall \triangle$. The justification of this bound is that $3 / 2$ is the maximum achievable value of $\delta_{\text {TтC }} /$ diam in standard hyperbolic space or in Euclidean space, while $\delta_{\text {ттс }} /$ diam goes beyond $3 / 2$ in positively curved spaces. (See [Jonckheere et al. 08] for the details.)

In this paper, we basically do the same analysis, but for the FPC. The fact that four instead of three points are involved leads to a greater variety of ways to scale $\delta$ than in the TTC case. Here, we consider the following scalings: $\delta(\square) / \operatorname{diam}(\square)$, $\delta(\square) / L(\square), \delta(\square) /(L+M+S)(\square)$, and compute the upper bound of such scaled 
$\delta$ 's in the standard Riemannian manifold $\mathbb{H}=\mathbb{M}_{-k^{2}}$ of constant negative curvature, in the Euclidean space $\mathbb{E}$, and in the standard manifold $\mathbb{S}=\mathbb{M}_{k^{2}}$ of constant positive curvature. In addition, because the scaled FPC relies on quadrilaterals instead of triangles as basic geometric objects, the suprema of the scaled $\delta$ 's can also be computed in Ptolemaic space $\mathbb{P}$ in a quite natural way. Furthermore, the recent reformulation of $\mathrm{CAT}(0)$ space in term of quadrilateral inequalities [Berg and Nikolaev 08] even allows us to compute the suprema of the scaled $\delta$ 's in $\mathrm{CAT}(0)$ space. To simplify the notation, the four scaled $\delta$ 's are denoted generically by $\delta / D$, where $D$ is any of the distance elements diam, $L, L+M+S$, or even $L-S$. We are now in a position to formulate our main result.

Theorem I.I. For the scalings $D=L, L+M+S$, and diam, the various $\delta / D$ 's behave as follows in the hyperbolic $(\mathbb{H})$, Euclidean $(\mathbb{E})$, Ptolemaic $(\mathbb{P}), \operatorname{CAT}(0)$, and spherical $(\mathbb{S})$ spaces:

$$
\begin{aligned}
& \sup _{\substack{a, b, c, d \in \mathbb{H} \\
d(i, j) \geq \epsilon>0, i \neq j}} \frac{\delta(a, b, c, d)}{D(a, b, c, d)} \\
& <\sup _{a, b, c, d \in \mathbb{H}} \frac{\delta(a, b, c, d)}{D(a, b, c, d)}=\sup _{a, b, c, d \in \mathbb{E}} \frac{\delta(a, b, c, d)}{D(a, b, c, d)}=\sup _{a, b, c, d \in \operatorname{CAT}(0)} \frac{\delta(a, b, c, d)}{D(a, b, c, d)} \\
& \quad \leq \sup _{a, b, c, d \in \mathbb{P}} \frac{\delta(a, b, c, d)}{D(a, b, c, d)}<\sup _{a, b, c, d \in \mathbb{S}} \frac{\delta(a, b, c, d)}{D(a, b, c, d)},
\end{aligned}
$$

where equality holds in the relation $\leq_{D}$ for $D=$ diam, and strict inequality holds for $D=L, L+M+S$. (See Table 1.) Furthermore, the Euclidean supremum is easily identified as being achieved for a 2-dimensional square. As for the $D=$ $L-S$ scaling, only the first inequality holds, since the others become equalities.

A few comments are in order if one is to understand the gist of the result. In the definition of the spaces $\mathbb{H}$ and $\mathbb{S}$, the curvature was set to $-k^{2}$ and $k^{2}$ across the respective spaces, but $k \neq 0$ was arbitrary. It is already a first observation that the suprema over $\mathbb{H}$ and $\mathbb{S}$ depend only on the sign of the curvature (see Section 4.3). The requirement $d(i, j) \geq \epsilon>0, i \neq j=a, b, c, d$, in the top supremum is to prevent it from being achieved for infinitesimally small distances among the four points, in which case the hyperbolic supremum coincides with the Euclidean one. We note that the diam scaling is a bit deficient, since it does not provide a distinction between, on the one hand, the spaces $\mathbb{H}, \mathbb{E}$, and $\operatorname{CAT}(0)$ and on the other hand, the space $\mathbb{P}$.

The overall string of (in)equalities is consistent with the TTC intuition that $\delta / D$ should be "small" in negative curvature and "bigger" in positive curvature 
[Jonckheere et al. 08]. We shall therefore declare a metric space to be scaled Gromov nonpositively curved if $\delta(a, b, c, d) / D(a, b, c, d)$ remains less than or equal to the Euclidean bound for all quadruples of points.

The $\delta / D$ analysis in various spaces was motivated by network problems (see Section 9.1), but the results raise the fundamental issue of what spaces $X$ are "between" the Euclidean and spherical spaces; precisely, $\sup _{\mathbb{E}} \delta / D<\sup _{X} \delta / D<$ $\sup _{\mathbb{S}} \delta / D$. As shown in this paper, the Ptolemaic space with the proper scaling is one such space, but whether there are other spaces within the discontinuity gap is widely open.

The scaled TTC analysis relied on a Cartan-Alexandrov-Toponogov (CAT) comparison argument [Jonckheere et al. 08]. Unfortunately, such a geometric approach does not appear to be workable for the FPC; therefore, here, we resort to a computational-algebraic approach: The above suprema are first computed numerically (see Sections 4,5 ). From the numerical values of the suprema in various spaces, we guess their exact values $\widehat{\delta / D}$, as well as the quadrilateral $\hat{\square}$ that achieves the optimum (see Section 6 ). Since the conditions for embeddability in Euclidean, $\mathrm{CAT}(0)$, and Ptolemaic spaces are purely algebraic, verifying that $\forall a, b, c, d, \delta(a, b, c, d) / D(a, b, c, d) \leq \widehat{\delta / D}$ is true and that $\exists(a, b, c, d): \delta(a, b, c, d) / D(a, b, c, d)>\widehat{\delta / D}$ is false should, in principle, be manageable via a Tarski-Seidenberg decision problem. Unfortunately, the Mathematica and MacAulay encodings of the preceding expressions together with the well-known Cayley-Menger, CAT(0), and Ptolemaic conditions for embeddability in the corresponding spaces result in running times of more than 24 hours, sometimes with saturated memories, without a decision being reached. ${ }^{1}$ However, a very recent reformulation of the $\mathrm{CAT}(0)$ conditions [Berg and Nikolaev 08] allows the Euclidean bound gleaned from numerical computation to be proved analytically (see Section 7). The Ptolemaic case, on the other hand, requires part of the quantifier elimination to be done "by hand" before Mathematica can positively confirm the bound. The latter part is a bit involved and therefore relegated to the appendix (Section 11).

The practical impact of this work is that comparison between the scaled 4point $\delta$ of an Internet graph and the bounds achievable in the various reference spaces for the various scalings allows us to model, in the spirit of coarse geometry, such network graphs as Riemannian manifolds, CAT(0), or even Ptolemaic spaces. In the case of a Riemannian manifold model, a finer classification is provided by the curvature, and it is along that important line of applications [Jonckheere et al. 11b] that the classical graph generators differ:

\footnotetext{
${ }^{1}$ The MacAulay encoding was done by Dr. Alex Shoshitaishvili.
} 
Major impact. Relative to the diameter scaling, and for a relevant combination of generator parameters, the standard graph generators (Barabási-Albert scalefree and $\beta$-model Watts-Strogatz small-world) behave as follows:

$$
\sup _{\square \subseteq\{\text { B-A scale-free }\}} \frac{\delta(\square)}{D(\square)} \approx \sup _{\square \subset \mathbb{H}} \frac{\delta(\square)}{D(\square)}<\sup _{\square \subseteq\{\text { W-S small-world }\}} \frac{\delta(\square)}{D(\square)}
$$

(see Figures 2 and 3). This conclusion is fully consistent with the TTC analysis [Jonckheere et al. 08], namely, that for some combination of the graph-generator parameters, scale-free graphs can be coarsely modeled as negatively curved Riemannian manifolds, and small-world graphs are modeled by positively curved manifolds.

\section{4-Point Inequality, 4-Point Condition}

Consider four points $a, b, c, d$ in a metric space $(X, d)$. As stated in the introduction, we need to consider all distances between all pairs of points. To simplify the notation, define

$$
\begin{aligned}
& u=d(a, d), v=d(b, c) ; \\
& x=d(a, b), y=d(c, d) ; \\
& z=d(a, c), w=d(b, d) ;
\end{aligned}
$$

with the assumption, incurring no loss of generality, that

$$
L:=u+v \geq M=: x+y \geq S:=z+w .
$$

Recall that an ultrametric space is a metric space in which the triangle inequality in, say, $\triangle a b c$ is strengthened to $v \leq \max \{x, z\}$ along with inequalities resulting from permutations of the sides of $\triangle a b c$. The significance of the concept is that $d$ is ultrametric iff it is the distance on an equidistant tree [Su 04, Theorem 6.1], that is, a tree that has constant distance between its root and its degree-one vertices. The metric space $(X, d)$ is said to satisfy the four-point inequality if it satisfies a quadrilateral version of the ultrametric condition. With our convention, the four-point inequality reduces to $L \leq \max \{M, S\}$, and further to $L=M$. It can be shown that a metric satisfies the four-point inequality if and only if it is the distance on a (not necessarily equidistant) tree [Su 04, Theorem 6.2]. Hence to make the space look like a tree at a large scale, the condition $L=M$ is relaxed to $L-M \leq 2 \delta$. Formally, we have the following definition. 
Definition 2.I. The metric space $(X, d)$ is said to satisfy the Gromov four-point condition $(F P C)$ if there exists $\bar{\delta}<\infty$ such that

$$
\sup _{a, b, c, d \in X} \overbrace{\frac{L(a, b, c, d)-M(a, b, c, d)}{2}}^{\delta(a, b, c, d)}<\bar{\delta} .
$$

\section{Algebraic and Trigonometric Characterization of Various Spaces}

In this section, various spaces are characterized in a way that is numerically, and sometimes analytically, tractable.

\section{I. Ptolemaic Spaces and the Cayley-Menger Matrix}

Definition 3.I. A metric space $(X, d)$ is said to be Ptolemaic if for any quadruple of points $\{a, b, c, d\} \subseteq X$, we have

$$
\begin{aligned}
& u v \leq x y+z w, \\
& x y \leq u v+z w, \\
& z w \leq u v+x y,
\end{aligned}
$$

where $u, \ldots, w$ are defined as in (2.1).

Ptolemy's theorem states that a quadruple of points on a Euclidean circle subject to the convention (2.2)) saturates the first inequality. Less trivial is the fact that the Euclidean space and the standard constant-negative-curvature manifold are Ptolemaic. One can generalize a bit further by saying that a $\operatorname{CAT}(0)$ space is Ptolemaic (but the converse is not true).

The Ptolemaic inequalities can be written in matrix format as follows: Define the "Ptolemaic matrix"

$$
P=\left(\begin{array}{cccc}
0 & x^{2} & z^{2} & u^{2} \\
x^{2} & 0 & v^{2} & w^{2} \\
z^{2} & v^{2} & 0 & y^{2} \\
u^{2} & w^{2} & y^{2} & 0
\end{array}\right) .
$$

Then it is easily established that

$$
\operatorname{det} P=(u v-x y-z w)(x y-u v-z w)(z w-x y-u v)(u v+x y+z w),
$$

and the Ptolemaic conditions are equivalent to $\operatorname{det} P \leq 0$. 
We now look at the more restrictive condition of embeddability in Euclidean space.

Theorem 3.2. There exists an isometric embedding $(\{a, b, c, d\}, d) \hookrightarrow \mathbb{E}^{r \leq 3}$, or equivalently, the edges of lengths $u, v, x, y, z, w$ form a complete Euclidean quadrilateral of $\mathbb{E}^{r}$, iff the Cayley-Menger matrix

$$
\mathrm{CM}=\left(\begin{array}{ccccc}
0 & 1 & 1 & 1 & 1 \\
1 & 0 & x^{2} & z^{2} & u^{2} \\
1 & x^{2} & 0 & v^{2} & w^{2} \\
1 & z^{2} & v^{2} & 0 & y^{2} \\
1 & u^{2} & w^{2} & y^{2} & 0
\end{array}\right)
$$

has a sequence of nested principal minors $\mathrm{CM}_{I \times I}, I \subseteq\{1,2,3,4,5\}$, starting at order $|I|=2$ with $\mathrm{CM}_{\{1,2\} \times\{1,2\}}=\left(\begin{array}{ll}0 & 1 \\ 1 & 0\end{array}\right)$ and running to order $r+2$ such that

$$
\operatorname{sign}\left(\operatorname{det} \mathrm{CM}_{I \times I}\right)=-(-1)^{|I|} \quad \text { for }|I| \leq r+2
$$

and

$$
\operatorname{det} \mathrm{CM}_{I \times I}=0 \quad \text { for }|I|>r+2 \text {. }
$$

Proof. See [Blumenthal 53, Theorems 41.1, 42.1].

Observe that for $|I|=3$, the sign constraints are completely trivial. For $|I|=4$, they yield the triangle inequalities; indeed, for $I=\{1,2,3,4\}$,

$$
\operatorname{det}\left(\begin{array}{cccc}
0 & 1 & 1 & 1 \\
1 & 0 & x^{2} & z^{2} \\
1 & x^{2} & 0 & v^{2} \\
1 & z^{2} & v^{2} & 0
\end{array}\right)=(x-v-z)(v-x-z)(z-x-v)(x+v+z)
$$

which is the triangle inequality for the subset of points $\{a, b, c\}$. Should the triangle inequality hold, then by Heron's theorem, the above is $-8(A(\triangle a b c))^{2}$. For $I=\{1,2,3,5\}$, det $\mathrm{CM}_{I \times I} \leq 0$ yields the triangle inequality for $\{a, b, d\}$; for $I=\{1,3,4,5\}$, the constraint yields the triangle inequality for $\{b, c, d\}$, etc. If, in addition, the last condition $\operatorname{det} \mathrm{CM} \geq 0$ holds, it is well known that $\operatorname{det} \mathrm{CM}=$ $288 \operatorname{vol}^{2}(\square a b c d)$, where $\operatorname{vol}(\square a b c d)$ is the volume of the tetrahedron with vertices 
$a, b, c, d$. Observe that

$\operatorname{det} \mathrm{CM}$

$$
\begin{aligned}
= & -2 x^{2} y^{4}-2 w^{4} z^{2}-2 v^{4} u^{2}-2 x^{4} y^{2}-2 z^{4} w^{2}-2 u^{4} v^{2} \\
& +2 v^{2} u^{2} y^{2}+2 w^{2} z^{2} y^{2}+2 x^{2} w^{2} y^{2}+2 w^{2} v^{2} u^{2}+2 x^{2} v^{2} y^{2}+2 z^{2} v^{2} u^{2} \\
& +2 v^{2} w^{2} z^{2}+2 x^{2} u^{2} y^{2}+2 x^{2} v^{2} u^{2}+2 x^{2} z^{2} y^{2}+2 x^{2} w^{2} z^{2}+2 z^{2} w^{2} u^{2} \\
& -2 v^{2} w^{2} y^{2}-2 x^{2} v^{2} z^{2}-2 x^{2} w^{2} u^{2}-2 z^{2} u^{2} y^{2} \\
= & 2\left(u^{2}+v^{2}\right)\left(x^{2} y^{2}-u^{2} v^{2}+z^{2} w^{2}\right)+2\left(x^{2}+y^{2}\right)\left(-x^{2} y^{2}+u^{2} v^{2}+z^{2} w^{2}\right) \\
& +2\left(z^{2}+w^{2}\right)\left(x^{2} y^{2}+u^{2} v^{2}-z^{2} w^{2}\right) \\
& -2 x^{2}\left(u^{2} w^{2}+z^{2} v^{2}\right)-2 y^{2}\left(v^{2} w^{2}+z^{2} u^{2}\right) .
\end{aligned}
$$

The case $I=\{2,3,4,5\}$ does not occur in a nested sequence of principal minors starting at $I=\{1,2\}$. Observe, however, that det $\mathrm{CM}_{\{2,3,4,5\} \times\{2,3,4,5\}} \leq 0$ is equivalent to the Ptolemaic conditions. By a fundamental congruence theorem, if there exists a nested sequence of principal minors of alternating signs, then $\operatorname{det} \mathrm{CM}_{\{2,3,4,5\} \times\{2,3,4,5\}} \leq 0$ and the Ptolemaic conditions hold.

It is trivial, but necessary, to observe that the Ptolemaic and Cayley-Menger conditions are scale-independent, in the sense that the various inequalities are preserved under a scaling of the form $(u, v, w, x, y, z) \mapsto(k u, k v, k w, k x, k y, k z)$, $k \in \mathbb{R}^{*}$.

\subsection{A Quadrilateral Inequality for $\operatorname{CAT}(0)$ Spaces}

As shown in the appendix, the Cayley-Menger conditions are difficult to manage in the realm of computer algebra. However, Berg and Nikolaev's new characterization of $\mathrm{CAT}(0)$ spaces via a quadrilateral inequality comes in handy as a replacement of the Cayley-Menger constraints (see [Berg and Nikolaev 08] and [Jost 97, Theorem 2.3.1] for a closely related result). The Berg and Nikolaev theorem states that any geodesic space $(X, d)$ is a $\mathrm{CAT}(0)$ space iff for any quadruple of points $a, b, c, d \in X$ we have

$$
d(a, d)^{2}+d(b, c)^{2} \leq d(a, b)^{2}+d(b, d)^{2}+d(d, c)^{2}+d(c, a)^{2},
$$

or equivalently,

$$
u^{2}+v^{2} \leq\left(x^{2}+y^{2}\right)+\left(z^{2}+w^{2}\right),
$$

along with similar inequalities for the other subsets of the partition.

\subsection{Gram Matrices}

Surprisingly, embeddability of a quadruple of points in a space of constant nonvanishing curvature is much easier than in the Euclidean case, since 
embeddability relies on Gram matrices. We define $M_{\kappa}^{r}$ to be the standard $r$ dimensional Riemannian manifold of constant curvature $\kappa$.

Theorem 3.3. There exists an isometric embedding $(\{a, b, c, d\}, d) \hookrightarrow M_{\kappa<0}^{r \leq 3}$ iff the Gram matrix

$$
G^{-}=\left(\begin{array}{cccc}
1 & \cosh (\sqrt{-\kappa} x) & \cosh (\sqrt{-\kappa} z) & \cosh (\sqrt{-\kappa} u) \\
\cosh (\sqrt{-\kappa} x) & 1 & \cosh (\sqrt{-\kappa} v) & \cosh (\sqrt{-\kappa} w) \\
\cosh (\sqrt{-\kappa} z) & \cosh (\sqrt{-\kappa} v) & 1 & \cosh (\sqrt{-\kappa} y) \\
\cosh (\sqrt{-\kappa} u) & \cosh (\sqrt{-\kappa} w) & \cosh (\sqrt{-\kappa} y) & 1
\end{array}\right)
$$

has a sequence of nested principal minors $G_{I \times I}^{-}, I \subseteq\{1,2,3,4\}$, starting at order $|I|=1$ and running to order $r+1$ such that

$$
\operatorname{sign}\left(\operatorname{det} G_{I \times I}^{-}\right)=-(-1)^{|I|} \text { for }|I| \leq r+1
$$

and

$$
\operatorname{det} G_{I \times I}^{-}=0 \quad \text { for }|I|>r+1 \text {. }
$$

Proof. See [Blumenthal 53, Theorem 106.1 and corollary].

Theorem 3.4. There exists an isometric embedding $(\{a, b, c, d\}, d) \hookrightarrow M_{\kappa>0}^{r \leq 3}$ iff $\operatorname{diam}\{a, b, c, d\} \leq \pi / \sqrt{\kappa}$ and the Gram matrix

$$
G^{+}=\left(\begin{array}{cccc}
1 & \cos (\sqrt{\kappa} x) & \cos (\sqrt{\kappa} z) & \cos (\sqrt{\kappa} u) \\
\cos (\sqrt{\kappa} x) & 1 & \cos (\sqrt{\kappa} v) & \cos (\sqrt{\kappa} w) \\
\cos (\sqrt{\kappa} z) & \cos (\sqrt{\kappa} v) & 1 & \cos (\sqrt{\kappa} y) \\
\cos (\sqrt{\kappa} u) & \cos (\sqrt{\kappa} w) & \cos (\sqrt{\kappa} y) & 1
\end{array}\right)
$$

is positive semidefinite of rank $(r+1)$, that is, if there exists a sequence of nested principal minors $G_{I \times I}^{+}, I \subseteq\{1,2,3,4\}$, starting at order $|I|=1$ and running to order $r+1$ such that

$$
\operatorname{sign}\left(\operatorname{det} G_{I \times I}^{+}\right)=+1 \quad \text { for }|I| \leq r+1
$$

and

$$
\operatorname{det} G_{I \times I}^{+}=0 \quad \text { for }|I|>r+1 \text {. }
$$

Proof. See [Blumenthal 53, Theorem 63.1]. 
Again, the sign constraints on $G^{ \pm}$for $|I|=1,2$ are completely trivial. For $|I|=3$, it is easy to see that because of the Gram nature of the matrices $G^{ \pm}$, the sign constraints are in fact triangle inequalities [Bridson and Haefliger 99].

More specifically, in the hyperbolic case, it is readily observed that

$$
\begin{aligned}
\operatorname{det} G_{\{1,2,3\} \times\{1,2,3\}}^{-}= & 1-\cosh ^{2}(\sqrt{-\kappa} v)-\cosh ^{2}(\sqrt{-\kappa} x)-\cosh ^{2}(\sqrt{-\kappa} z) \\
& +2 \cosh (\sqrt{-\kappa} v) \cosh (\sqrt{-\kappa} x) \cosh (\sqrt{-\kappa} z) .
\end{aligned}
$$

By the hyperbolic Heron formula, the condition $\operatorname{det} G_{\{1,2,3\} \times\{1,2,3\}}^{-} \geq 0$ is equivalent to the triangle inequality in $\triangle a b c$ and

$$
\begin{aligned}
& \operatorname{det} G_{\{1,2,3\} \times\{1,2,3\}}^{-} \\
& \quad=\left((1+\cosh (\sqrt{-\kappa} v)+\cosh (\sqrt{-\kappa} x)+\cosh (\sqrt{-\kappa} z)) \tan \left(\frac{A(\triangle a b c)}{2}\right)\right)^{2} .
\end{aligned}
$$

In the spherical case, $\operatorname{det} G_{\{1,2,3\} \times\{1,2,3\}}^{+} \geq 0$ is related to $A(\triangle a b c)$, and the latter is related to the triangle inequalities via L'Huilier's formula:

$$
\tan ^{2} \frac{A(\triangle a b c)}{4}=\tan \frac{x+z+v}{4} \tan \frac{x+z-v}{4} \tan \frac{x+v-z}{4} \tan \frac{z+v-y}{4} .
$$

The highest-order condition $\operatorname{det} G^{ \pm} \geq 0$ is related to the volume.

Specifically, if the last condition $\operatorname{det} G^{-} \geq 0$ holds, then $\operatorname{det} G^{-}$is related to $\operatorname{vol}(\square a b c d)$, but not via an easy formula [Murakami and Ushijima 05, Theorem 2.2], from which it nevertheless follows that

$$
\operatorname{det} G^{+}=0 \Longrightarrow \operatorname{vol}(\square a b c d)=0 .
$$

The Gram matrix conditions are a bit different from the Ptolemaic inequality and the Cayley-Menger conditions in that they are scale-independent under a rescaling of the curvature. Specifically, if $(u, v, x, y, z, w)$ is embeddable in $M_{\kappa}$, then $(k u, k v, k x, k y, k z, k w), k>0$, is embeddable in $M_{\kappa / k^{2}}$.

If the Gram matrix is singular, then scale-independence holds only under rescaling of the curvature. As a counterexample, consider two equilateral triangles $\triangle a b c, \triangle d b c \subset M_{-1}$, where $d(a, b)=d(a, c)=d(b, c)=d(b, d)=d(c, d)=1$; the two triangles are glued along their common side $[b, c]$ such that the common foot $h$ of the altitudes $[a, h],[d, h]$ is "between" $a$ and $d$. The later "betweenness" concept means that $d(a, d)=d(a, h)+d(h, d)$. The internal angle of the equilateral triangle is

$$
\alpha=\cos ^{-1} \frac{\cosh (1)^{2}-\cosh (1)}{\sinh (1)^{2}} \approx 0.9188 .
$$

The length of the altitude is $\ell([a, h])=\sinh ^{-1}(\sin (\alpha) \sinh (1)) \approx 0.8340$. Hence $d(a, d) \approx 1.668$. With this system of distances, the principal minors of the Gram 
matrix are $(1.0,-1.3810978,1.205158,-0.0)$, with the last one vanishing, as expected. However, if we amplify those distances by a factor $k>1$, the last minor of the Gram matrix becomes positive. Hence the system of points is no longer isometrically embeddable in the hyperbolic space $\mathbb{M}_{-1}$; the only space in which it is isometrically embeddable is $M_{-1 / k^{2}}$.

\section{Generic Constrained Optimization Problem}

Finding the upper bounds on the Gromov 4-point $\delta$ for the various spaces is basically the constrained optimization problem

$$
\sup _{a, b, c, d} \frac{\delta(a, b, c, d)}{D(a, b, c, d)}
$$

subject to the constraint that the quadruple $\{a, b, c, d\}$ be isometrically embeddable in the specific space: negatively curved, Euclidean, Ptolemaic, positively curved. Since embeddability in the various spaces is expressed in terms of the distances, the problem is conveniently reformulated in terms of those distances,

$$
\sup _{u, v, x, y, z, w} \frac{L(u, v, x, y, z, w)-M(u, v, x, y, z, w)}{2 D(u, v, x, y, z, w)}
$$

subject to various constraints:

Linear constraints: to enforce the fundamental triangle inequalities $L \geq M \geq$ $S$ and other convenient assumptions incurring no loss of generality. In addition, in the diameter scaling case, we enforce $u$ to be the diameter.

Nonlinear constraints: to enforce the Ptolemaic inequalities and the various sign constraints on the principal minors of the Cayley-Menger and Gram matrices.

\section{I. Linear Constraints}

The $3\left(\begin{array}{l}4 \\ 3\end{array}\right)=12$ triangle inequalities can conveniently be written as

$$
\begin{array}{ll}
u \leq \min \{x+w, z+y\}, & v \leq \min \{x+z, w+y\}, \quad x \leq \min \{z+v, u+w\} \\
y \leq \min \{v+w, u+z\}, & z \leq \min \{u+y, v+x\}, \quad w \leq \min \{v+y, u+x\}
\end{array}
$$

If we define

$$
\xi=(u, v, x, y, z, w)^{\prime},
$$

the triangle inequalities can be rewritten as $A_{t} \xi \leq 0$, where $A_{t}$ is a $12 \times 6$ matrix. 
The constraint $L \geq M \geq S$ can be rewritten as $A_{\mathrm{LMS}} \xi \leq 0$, where $A_{\mathrm{LMS}}$ is a $2 \times 6$ matrix.

The convenient linear constraints, incurring no loss of generality, are as follows:

$$
u \geq v, \quad x \geq y, \quad z \geq w .
$$

They are convenient because they restrict the diameter to $u, x$, or $z$. Again, they can be rewritten as $A_{\text {wlog }} \xi \leq 0$, where $A_{\text {wlog }}$ is a $3 \times 6$ matrix.

The linear constraints are written compactly as $A \xi \leq 0$, where

$$
A=\left(A_{t}^{\prime}, A_{\text {LMS }}^{\prime}, A_{\text {wlog }}^{\prime}\right)^{\prime} .
$$

Specifically,

$$
\begin{aligned}
A_{t} & =\left(\begin{array}{rrrrrr}
1 & 0 & -1 & 0 & 0 & -1 \\
1 & 0 & 0 & -1 & -1 & 0 \\
0 & 1 & -1 & 0 & -1 & 0 \\
0 & 1 & 0 & -1 & 0 & -1 \\
0 & -1 & 1 & 0 & -1 & 0 \\
-1 & 0 & 1 & 0 & 0 & -1 \\
0 & -1 & 0 & 1 & 0 & -1 \\
-1 & 0 & 0 & 1 & -1 & 0 \\
-1 & 0 & 0 & -1 & 1 & 0 \\
0 & -1 & -1 & 0 & 1 & 0 \\
0 & -1 & 0 & -1 & 0 & 1 \\
-1 & 0 & -1 & 0 & 0 & 1
\end{array}\right), \\
A_{\mathrm{LMS}} & =\left(\begin{array}{rrrrrr}
-1 & -1 & 1 & 1 & 0 & 0 \\
0 & 0 & -1 & -1 & +1 & +1
\end{array}\right), \\
A_{\mathrm{w} \log } & =\left(\begin{array}{rrrrrrr}
-1 & 1 & 0 & 0 & 0 & 0 \\
0 & 0 & -1 & 1 & 0 & 0 \\
0 & 0 & 0 & 0 & -1 & 1
\end{array}\right) .
\end{aligned}
$$

\subsection{Nonlinear Constraints}

The nonlinear constraints in the various spaces involve either trigonometric or polynomial inequalities $c_{i}(\xi) \leq 0$, with the extra requirement that if one of the inequalities saturates, others might have to saturate as well. The precise sign requirement is written as $c(\xi) \unlhd 0$, to indicate that the embeddability conditions are more stringent than just $c(\xi) \leq 0$. 
The nonlinear constraints in a Ptolemaic space are written either as the three inequalities $(3.1)$, to be written $c_{1}(\xi) \leq 0, c_{2}(\xi) \leq 0, c_{3}(\xi) \leq 0$, or as the single, but higher-degree, inequality $\operatorname{det} P:=c_{1} \leq 0$, where $\operatorname{det} P$ is given by (3.2). There are no restrictions on the saturation; nevertheless, we keep the notation $c(\xi) \unlhd 0$.

The new formulation of $\mathrm{CAT}(0)$ spaces involves quadrilateral inequalities, again without restrictions on the saturation; nevertheless, we keep the notation $c(\xi) \unlhd 0$.

Regarding the nonlinear constraints of the Euclidean case, those corresponding to determinants of order $|I|=\operatorname{size}(\mathrm{CM})-1=4$ of the relevant Cayley-Menger matrix involve the product form of the three triangle inequalities in all four triangles of the quadrilateral; the latter are written $c_{i}(\xi) \leq 0, i=1,2,3$, 4, where, for example, $c_{1}$ is given by (3.3). Should any of them vanish, then the determinant of the full matrix $\operatorname{det} \mathrm{CM}=: c_{5}$ has to vanish, in which case the quadruple is embeddable in a subspace of dimension $\leq 2$. Therefore, the constraints can be formalized as $C_{1} \vee C_{2} \vee C_{3}$, where

$$
\begin{aligned}
& C_{1}=\left(c_{1}<0\right) \wedge\left(c_{2}<0\right) \wedge\left(c_{3}<0\right) \wedge\left(c_{4}<0\right) \wedge\left(c_{5}<0\right), \\
& C_{2}=\left(c_{1}<0\right) \wedge\left(c_{2}<0\right) \wedge\left(c_{3}<0\right) \wedge\left(c_{4}<0\right) \wedge\left(c_{5}=0\right), \\
& C_{3}=\left(\forall i \in\{1,2,3,4\}, c_{i} \leq 0\right) \wedge\left(\exists i \in\{1,2,3,4\}: c_{i}=0\right) \wedge\left(c_{5}=0\right) .
\end{aligned}
$$

We write $C_{1} \vee C_{2} \vee C_{3}$ as $c(\xi) \unlhd 0$.

For the Riemannian cases of negative and positive curvature, the constraints on the principal minors of order $|I|=\operatorname{size}\left(G^{ \pm}\right)-1=3$ are triangle inequalities, to be written $c_{i}(\xi) \leq 0, i=1,2,3,4$. The condition on the full matrix is written $c_{5}(\xi) \leq 0$. Again, one has to be cautious, since if one of the triangle inequalities saturates, i.e., $c_{i}(\xi)=0$ for some $i \in\{1,2,3,4\}$, then the fifth inequality saturates as well, i.e., $c_{5}(\xi)=0$, meaning that the volume vanishes. Therefore, the constraints can be formalized as $C_{1} \vee C_{2} \vee C_{3}$, where

$$
\begin{aligned}
& C_{1}=\left(c_{1}<0\right) \wedge\left(c_{2}<0\right) \wedge\left(c_{3}<0\right) \wedge\left(c_{4}<0\right) \wedge\left(c_{5}<0\right), \\
& C_{2}=\left(c_{1}<0\right) \wedge\left(c_{2}<0\right) \wedge\left(c_{3}<0\right) \wedge\left(c_{4}<0\right) \wedge\left(c_{5}=0\right), \\
& C_{3}=\left(c_{i} \leq 0, \forall i \in\{1,2,3,4\}\right) \wedge\left(\exists i \in\{1,2,3,4\}: c_{i}=0\right) \wedge\left(c_{5}=0\right) .
\end{aligned}
$$

We write $C_{1} \vee C_{2} \vee C_{3}$ as $c(\xi) \unlhd 0$.

\subsection{Scale Independence}

Proposition 4.I. $\sup _{a, b, c, d \in M_{\kappa \neq 0}} \frac{\delta(a b c d)}{D(a b c d)}$ depends only on the sign of the curvature, not on its magnitude. 
Proof. Let $c_{\kappa}(\xi) \unlhd 0$ denote nonlinear embeddability constraints in $M_{\kappa \neq 0}$. Let $\kappa^{\prime}$ be another curvature (of the same sign). It is easily verified that $c_{\kappa^{\prime}}(\xi)=c_{\kappa}\left(\sqrt{\frac{\kappa^{\prime}}{\kappa}} \xi\right)$. Define $\xi^{\prime}=\sqrt{\frac{\kappa^{\prime}}{\kappa}} \xi$. We have

$$
\sup _{A \xi \leq 0, c_{\kappa^{\prime}}(\xi) \unlhd 0} \frac{\delta(\xi)}{D(\xi)}=\sup _{A \xi^{\prime} \leq 0, c_{\kappa}\left(\xi^{\prime}\right) \unlhd 0} \frac{\delta\left(\sqrt{\frac{\kappa}{\kappa^{\prime}}} \xi^{\prime}\right)}{D\left(\sqrt{\frac{\kappa}{\kappa^{\prime}}} \xi^{\prime}\right)}=\sup _{A \xi^{\prime} \leq 0, c_{\kappa}\left(\xi^{\prime}\right) \unlhd 0} \frac{\delta\left(\xi^{\prime}\right)}{D\left(\xi^{\prime}\right)},
$$

where the last equality stems from the trivial scale invariance of $\delta / D$. The result follows from the extreme sides of the above equality.

Proposition 4.2. In the Ptolemaic, Euclidean, and $\mathrm{CAT}(0)$ cases, if $\hat{\xi}$ reaches

$$
\sup _{A \xi \leq 0, c(\xi) \unlhd 0} \frac{\delta(\xi)}{D(\xi)},
$$

so does $k \hat{\xi}, k>0$. In the $\mathbb{M}_{\kappa \neq 0}$ case, if $\hat{\xi}$ reaches

$$
\sup _{A \xi \leq 0, c_{\kappa}(\xi) \unlhd 0} \frac{\delta(\xi)}{D(\xi)},
$$

then $k \hat{\xi}, k>0$, reaches

$$
\sup _{A \xi \leq 0, c_{\kappa / k^{2}}(\xi) \unlhd 0} \frac{\delta(\xi)}{D(\xi)}
$$

Proof. The first part is trivial from the homogeneous property of the Ptolemaic, Cayley-Menger, and quadrilateral inequality CAT(0) conditions. The second part results from manipulation of the arguments of cos and cosh in the Gram matrix conditions.

The scaling issue in negatively curved manifolds is different from that in positively curved manifolds. In the standard positively curved space, it is tacitly assumed that $u, v, x, y, z, w \leq \pi / \sqrt{\kappa}$. In hyperbolic space, the supremum is achieved for an infinitesimally small quadrilateral. Precisely, we have the following result.

Proposition 4.3.

$$
\lim _{\epsilon \downarrow 0} \sup _{\substack{a, b, c, d \in \mathbb{M}^{k<0} \\ u, v, x, y, z, w \geq \epsilon}} \frac{\delta(a, b, c, d)}{D(a, b, c, d)}=\sup _{\substack{a, b, c, d \in \mathbb{M}_{0} \\ u, v, x, y, z, w \geq \epsilon}} \frac{\delta(a, b, c, d)}{D(a, b, c, d)} .
$$

Proof. Indeed, the $1 \times 1$ and $2 \times 2$ conditions on the Gram $G^{-}$matrix are trivial, as are the $2 \times 2$ and $3 \times 3$ conditions on the Cayley-Menger CM matrix. Next, 
the $3 \times 3$ conditions on $G^{-}$are equivalent to the triangle inequalities, as are the $4 \times 4$ conditions on $\mathrm{CM}$, except for the $\{2,3,4,5\} \times\{2,3,4,5\}$ condition on $\mathrm{CM}$, which is equivalent to the Ptolemaic conditions. Hence all that remains to be proved is the equivalence between the $4 \times 4 G^{-}$condition at small scale and the $5 \times 5 \mathrm{CM}$ condition. Precisely, the proof relies on the observation that the $4 \times 4$ Gram $G^{-}$condition, up to eighth order, is equivalent to the $5 \times 5$ Cayley-Menger conditions. Clearly,

$$
G^{-}=\left(\begin{array}{cccc}
1 & 1+\frac{x^{2}}{2} & 1+\frac{z^{2}}{2} & 1+\frac{u^{2}}{2} \\
1+\frac{x^{2}}{2} & 1 & 1+\frac{v^{2}}{2} & 1+\frac{w^{2}}{2} \\
1+\frac{z^{2}}{2} & 1+\frac{v^{2}}{2} & 1 & 1+\frac{y^{2}}{2} \\
1+\frac{u^{2}}{2} & 1+\frac{w^{2}}{2} & 1+\frac{y^{2}}{2} & 1
\end{array}\right)+0\left(\xi^{2}\right)
$$

The determinant of the second-order component of $G^{-}$is found to be

$$
\begin{aligned}
\frac{1}{16}( & -2 x^{2} v^{2} u^{2} y^{2}-2 x^{2} w^{2} z^{2} y^{2}-2 z^{2} w^{2} v^{2} u^{2}+x^{4} y^{4}+z^{4} w^{4}+u^{4} v^{4}+4 z^{4} w^{2} \\
& +4 u^{4} v^{2}+4 v^{4} u^{2}+4 x^{4} y^{2}+4 w^{4} z^{2}+4 x^{2} y^{4}-4 x^{2} w^{2} z^{2}-4 z^{2} w^{2} u^{2} \\
& -4 v^{2} u^{2} y^{2}-4 w^{2} z^{2} y^{2}-4 x^{2} v^{2} u^{2}-4 x^{2} z^{2} y^{2}-4 x^{2} w^{2} y^{2}-4 w^{2} v^{2} u^{2} \\
& -4 z^{2} v^{2} u^{2}-4 x^{2} v^{2} y^{2}-4 v^{2} w^{2} z^{2}-4 x^{2} u^{2} y^{2}+4 x^{2} w^{2} u^{2}+4 z^{2} u^{2} y^{2} \\
& \left.+4 x^{2} v^{2} z^{2}+4 v^{2} w^{2} y^{2}\right)
\end{aligned}
$$

It is easily seen that the sixth-order term of the above is exactly $-\frac{1}{8} \operatorname{det}$ CM.

\section{Numerical Results}

The problem is set up, conceptually as follows:

$$
\sup _{\substack{A \xi \leq 0 \\ c(\xi) \unlhd 0}} \frac{L(\xi)-M(\xi)}{2 D(\xi)},
$$

where $A \xi \leq 0$ are the linear constraints and $c(\xi) \unlhd 0$ are the nonlinear constraints. It is good to recall that $L(\cdot), M(\cdot)$, and $D(\cdot)$ are linear.

The routine fmincon of Matlab is used to find the solution to the constrained optimization problem. The initial estimate $\xi_{0}$ is taken to be the solution to the problem with linear constraints only, which reduces to a (computationally reliable!) linear programming problem. Indeed, $m=\sup _{A \xi \leq 0} \frac{L-M}{2 D}$ can be rewritten as $\inf _{A \xi \leq 0}(2 D m-(L-M))=0$. The latter is a linear programming problem that can be iterated on $m$ using the Matlab routine linprog until a vanishing minimum is reached. 


\section{I. Linear Programming Results}

The maxima of $\frac{L-M}{2 D}$ subject to the linear constraints only for the various scalings are tabulated in the following table:

\begin{tabular}{c|c}
$D$ & $\sup _{A \xi \leq 0} \frac{\delta}{D}$ \\
\hline$L$ & 0.25 \\
$L+M+S$ & 0.125 \\
$\operatorname{diam}$ & 0.5 \\
$L-S$ & 0.5
\end{tabular}

In fact, these numerical results can be confirmed analytically.

Theorem 5.I. Consider the linear programming problem

$$
\min _{A \xi \leq 0}(2 m D-(L-M)) \text {. }
$$

For $D=L, \forall m$, the solution is

$$
(u, v, x, y, z, w)=\rho(2,2,1,1,1,1),
$$

and the optimal cost vanishes for $m=0.25$. For $D=L+M+S$, the solution is the same, provided $m \leq 0.125$, and the optimal cost vanishes for $m=0.125$. For $D=$ diam, the optimal solution is still the same $\forall m$ and the optimal cost vanishes for $m=0.5$. Finally, for $D=L-S$, the optimal cost vanishes for $m=0.5$.

Proof. For transparency of the proof, we discard the constraint $A_{\mathrm{w} \log } \xi \leq 0$, since it is not really necessary, and set $A=\left(A_{t}^{\prime} A_{\mathrm{LMS}}^{\prime}\right)^{\prime}$. We first follow the path that the proofs of the $D=L, D=L+M+S, D=L-S$, and $D=$ diam cases have in common. The inequality constraint $A \xi \leq 0$ is rewritten as an equality constraint $\sigma+A \xi=0$, where $\sigma \geq 0$ is a vector of slack variables. Next, it is necessary to impose an upper bound on the solution $\xi$, for otherwise it is infinite. Again, this is done by means of another slack vector $\tau \geq 0$, and the upper bound $\xi \leq 2$ is rewritten as another equality constraint $\tau+\xi=2 e$, where $e=\left(\begin{array}{llll}1 & 1 & \ldots\end{array}\right)^{\prime}$. Augmenting the state vector as $\left(\sigma^{\prime} \tau^{\prime} \xi^{\prime}\right)^{\prime}$, the constraints can be rewritten as

$$
\left(\begin{array}{lll}
I & 0 & A \\
0 & I & I
\end{array}\right)\left(\begin{array}{l}
\sigma \\
\tau \\
\xi
\end{array}\right)=\left(\begin{array}{l}
0 \\
2
\end{array}\right) .
$$

Furthermore, if the cost is written as $2 m D-(L-M)=\gamma \xi$, the linear programming tableau is 


\begin{tabular}{c|c||c||c}
$I_{14 \times 14}$ & $0_{14 \times 6}$ & $A_{14 \times 6}$ & $0_{14 \times 1}$ \\
\hline $0_{6 \times 14}$ & $I_{6 \times 6}$ & $I_{6 \times 6}$ & $2_{6 \times 1}$ \\
\hline \hline $0_{1 \times 14}$ & $0_{1 \times 6}$ & $\gamma_{1 \times 6}$ & 0
\end{tabular}

Essentially, we set $m$ to its optimum value, and endeavor to show that the minimum of $\gamma \xi$ is indeed 0 for $\xi=(2,2,1,1,1,1)$. It is trivial to verify that $\xi=(2,2,1,1,1,1)$ satisfies the constraints and that $\gamma \xi=0$. The nontrivial step is to show that $\xi=(2,2,1,1,1,1)$ is indeed the optimum. This is accomplished via a pivoting procedure on the above tableau. As the original tableau stands, the basic feasible solution is $\left(\sigma^{\prime}, \mu^{\prime}, \xi^{\prime}\right)^{\prime}=\left(0^{\prime}, 2^{\prime}, 0^{\prime}\right)^{\prime}$. Since all $\xi$ variables have to be activated, we move the $\left(\begin{array}{c}A \\ I \\ \gamma\end{array}\right)$ part of the tableau across the double vertical line to the extreme left of the tableau. To compensate for this, six among the $\sigma$ and $\tau$ slack columns have to be moved across the double vertical line to the right, where they will become vanishing, hence saturating some constraints. From the presumed solution, it is easily seen that the constraints to be saturated are those corresponding to $\sigma_{\{1,2,3,4,14\}}$ and $\tau_{1}$. Indeed, $\sigma_{\{1,2,3,4\}}=0$ yields saturation of the first four triangle inequalities $u \leq x+w, u \leq z+y, v \leq x+z, v \leq w+y$; $\sigma_{14}=0$ yields saturation of $M \geq S ; \tau_{1}=0$ yields saturation of $u \leq 2$. Regarding saturation of $v \leq 2$, the latter is equivalent to $\tau_{2}=0$, which, as we will soon see, comes out of the new basic feasible solution. After this operation, the tableau becomes

\begin{tabular}{c|c|c||c|c|c||c}
$A_{14 \times 6}$ & $\begin{array}{c}0_{4 \times 9} \\
I_{9 \times 9} \\
0_{1 \times 9}\end{array}$ & $0_{14 \times 5}$ & $\begin{array}{c}I_{4 \times 4} \\
0_{10 \times 4}\end{array}$ & $\begin{array}{c}0_{13 \times 1} \\
1\end{array}$ & $0_{14 \times 1}$ & $0_{14 \times 1}$ \\
\hline$I_{6 \times 6}$ & $0_{6 \times 9}$ & $\begin{array}{c}0_{1 \times 5} \\
I_{5 \times 5}\end{array}$ & $0_{6 \times 4}$ & $0_{6 \times 1}$ & $\begin{array}{c}1 \\
0_{5 \times 1}\end{array}$ & $2_{6 \times 1}$ \\
\hline \hline$\gamma$ & $0_{1 \times 9}$ & $0_{1 \times 5}$ & $0_{1 \times 4}$ & 0 & 0 & $0_{1 \times 1}$
\end{tabular}

We rewrite the above tableau in a more compact format as

\begin{tabular}{c||c||c}
$A_{1}$ & $A_{2}$ & $b$ \\
\hline \hline$\gamma$ & 0 & ||$c$
\end{tabular}

By row operations, we convert $A_{1}$ to the identity matrix, so as to obtain the near-canonical form

$$
\begin{array}{c||c||c}
I_{20 \times 20} & A_{1}^{-1} A_{2} & A_{1}^{-1} b \\
\hline \hline \gamma & 0 & 0
\end{array}
$$

To obtain the canonical form, we reduce $\gamma$ to 0 by trivial row operations to obtain

$$
\begin{array}{c||c||c}
I_{20 \times 20} & A_{1}^{-1} A_{2} & A_{1}^{-1} b \\
\hline \hline 0 & c_{2} & 0
\end{array}
$$


It follows from the above that the new basic feasible solution is

$$
\left(\xi^{\prime} \sigma_{5, \ldots, 13}^{\prime} \tau_{2, \ldots, 6}^{\prime}\right)^{\prime}=A_{1}^{-1} b .
$$

It is also easily observed that $\left(A_{1}^{-1} b\right)_{16}=\tau_{2}=0$; as expected, the constraint $v \leq 2$ is saturated. Since the $(2,3)$-block element of the above tableau vanishes, the cost vanishes for the basic feasible solution.

It remains to prove that this solution is optimal. For $D=L$, we have

$$
\gamma=\left(\begin{array}{llllll}
-1 & -1 & 2 & 2 & 0 & 0
\end{array}\right)
$$

and it follows that

$$
c_{2}=\left(\begin{array}{llllll}
1 / 2 & 1 / 2 & 1 / 2 & 1 / 2 & 1 & 0
\end{array}\right) .
$$

Since those relative cost coefficients are nonnegative, optimality is proved [Luenberger 89 , Section 3.4]. For the case $D=L+M+S$, we have

$$
\gamma=\left(\begin{array}{llllll}
-3 & -3 & 5 & 5 & 1 & 1
\end{array}\right)
$$

and it follows that

$$
c_{2}=\left(\begin{array}{llllll}
3 / 2 & 3 / 2 & 3 / 2 & 3 / 2 & 2 & 0
\end{array}\right) .
$$

Since those coefficients are nonnegative, optimality is proved as well. For $D=$ $L-S$, we have $\gamma=\left(\begin{array}{llllll}0 & 0 & 1 & 1 & -1 & -1\end{array}\right)$, and it follows that

$$
c_{2}=\left(\begin{array}{llllll}
0 & 0 & 0 & 0 & 1 & 0
\end{array}\right) .
$$

For $D=$ diam, we have $\gamma=\left(\begin{array}{llllll}0 & -1 & 1 & 1 & 0 & 0\end{array}\right)$, and it follows that

$$
c_{2}=\left(\begin{array}{llllll}
0 & 0 & 1 / 2 & 1 / 2 & 1 / 2 & 0
\end{array}\right) .
$$

The fact that in all scaling cases, some components of $c_{2}$ vanish reveals the possibility of multiple optima. (Recall that the vanishing of some relative cost coefficient is necessary but not sufficient for multiple optimal solutions [Appa 02].)

Lemma 5.2. Consider the linear programming problem $\min _{\bar{A} \chi=\bar{b}} \bar{\gamma} \chi=0$. Define the associated linear programming problems

$$
\operatorname{lp}_{k}: \min _{\bar{A}_{k} \chi_{k}=\bar{b}_{k}} \bar{\gamma}_{k} \chi_{k}, \quad k=0,1, \ldots
$$

The linear programs are initialized as

$$
\bar{\gamma}_{0}=\bar{\gamma}, \quad \bar{A}_{0}=\bar{A}, \quad \bar{b}_{0}=\bar{b} .
$$


In this particular application, the initialization is given as

$$
\begin{aligned}
& \bar{\gamma}_{0}=\bar{\gamma}=\left(\begin{array}{lll}
0 & 0 & \gamma
\end{array}\right), \\
& \bar{A}_{0}=\bar{A}=\left(\begin{array}{lll}
I & 0 & A \\
0 & I & I
\end{array}\right), \\
& \bar{b}_{0}=\bar{b}=\left(\begin{array}{c}
0_{14 \times 1} \\
2_{6 \times 1}
\end{array}\right) .
\end{aligned}
$$

Recursively, if $\chi_{k-1}^{*}$ is a solution to $\operatorname{lp}_{k-1}$ and as long as $\bar{\gamma}_{k-1} \chi_{k-1}^{*}<0$, the program $\operatorname{lp}_{k}$ is defined as follows:

$$
\bar{A}_{k}=\left(\begin{array}{c}
\bar{A}_{k-1} \\
\bar{\gamma}_{k-1}
\end{array}\right), \quad \bar{b}_{k}=\left(\begin{array}{c}
\bar{b}_{k-1} \\
\bar{\gamma}_{k-1} \chi_{k-1}^{*}
\end{array}\right),
$$

and the cost coefficients are defined as

$$
\left(\bar{\gamma}_{k}\right)_{i}= \begin{cases}-1 & \text { if }\left(\chi_{k-1}^{*}\right)_{i}=0 \\ 0 & \text { otherwise. }\end{cases}
$$

Then the solution $\chi_{k-1}^{*}, k=1,2, \ldots$, is unique iff $\bar{\gamma}_{k} \chi_{k}^{*}=0$.

Proof. The first step $k=1$ is proved in [Appa 02]. If $\bar{\gamma}_{1} \chi_{1}=0$, the optimal solution $\chi_{0}^{*}$ is unique, and the algorithm terminates. If $\bar{\gamma}_{1} \chi_{1}^{*} \neq 0$, the $\chi_{0}^{*}$ solution is nonunique, since $\chi_{1}$ is another solution. But the question now is whether besides $\chi_{0,1}$ there are still other solutions.

Then we use again the results of [Appa 02] to check whether the solution to $l p_{1}$ is unique. This defines the problem $\operatorname{lp}_{2}$, which sets the stage for the recursion. Clearly the recursion stops when $\bar{\gamma}_{k} \chi_{k}=0$.

For $D=L$, the first iteration of the algorithm of Lemma 5.2 yields

$$
\chi_{1}=\left(\begin{array}{llll}
0_{1 \times 12} & 0_{1 \times 2} & 2_{1 \times 6} & 0_{1 \times 6}
\end{array}\right)^{\prime}
$$

along with $\bar{\gamma}_{1} \chi_{1}=-4$, so that the $\chi$-solution is nonunique. Even though the $\xi$-component vanishes, it is necessary to run the iteration at least one more time, for there is no way to rule out the next solution having a nonvanishing $\xi$-component, hence revealing another $\xi$-solution. The next iteration yields

$$
\chi_{2}=\left(\begin{array}{llll}
0_{1 \times 4} \mid 0.3077_{1 \times 8} & 0 \mid 0.3077 & 1.6923_{1 \times 2} \mid 1.8461_{1 \times 4} & 0.3077_{1 \times 2} \mid 0.1539_{1 \times 4}
\end{array}\right)^{\prime} .
$$

Clearly the $\xi$-component is of the form $\rho(2,2,1,1,1,1)$. From here on, the $\xi$ solution cycles. Hence the $\xi=(2,2,1,1,1,1)$ solution is unique, up to a multiplicative positive constant. 
For $D=L+M+S$, the first iteration yields

$$
\chi_{1}=\left(\begin{array}{llll}
0_{1 \times 12} & 0_{1 \times 2} & 2_{1 \times 6} & 0_{1 \times 6}
\end{array}\right)^{\prime}
$$

along with $\bar{\gamma}_{1} \chi_{1}=-4$, so that the $\chi$-solution is nonunique. The situation is much the same as the previous case. The next iteration yields

$$
\chi_{2}=\left(\begin{array}{llll}
0_{1 \times 4} \mid 0.3077_{1 \times 8} & 0 \mid 0.3077 & 1.6923_{1 \times 2} \mid 1.8461_{1 \times 4} & 0.3077_{1 \times 2} \mid 0.1538_{1 \times 4}
\end{array}\right) .
$$

As in the preceding case, the $\xi=(2,2,1,1,1,1)$-solution is unique up to a multiplicative positive factor.

As for $D=L-S$, the first iteration of the algorithm of Lemma 5.2 yields

$$
\chi_{1}=\left(\begin{array}{llll}
2_{1 \times 12} & 0_{1 \times 2} & 0_{1 \times 6} & 2_{1 \times 6}{ }^{\prime}
\end{array}\right.
$$

along with $\bar{\gamma}_{1} \chi_{1}=-8$, so that the $\chi$-solution is nonunique, and more importantly, the $\xi$ part of the $\chi$ solution is nonunique. This component, in fact, yields an alternative solution that will prove useful in the nonlinear part of the algorithm. However, running another iteration yields

$\chi_{2}=\left(\begin{array}{llll}0.4690_{1 \times 4} \mid 0.8643_{1 \times 8} & 0 \mid 0.3953 & 1.357_{1 \times 2} \mid 1.3333_{1 \times 4} & 0.8643_{1 \times 2} \mid 0.6667_{1 \times 4}\end{array}\right)^{\prime}$.

Clearly, this reveals another $\xi$ solution,

$$
\xi=(0.8643,0.8643,0.6667,0.6667,0.6667,0.6667) .
$$

The next iteration does not have feasible solution. Hence all $\xi$ solutions are positive combinations of

$(2,2,1,1,1,1), \quad(2,2,2,2,2,2), \quad(0.8643,0.8643,0.6667,0.6667,0.6667,0.6667)$.

\subsection{Nonlinear Programming Results}

The preceding linear constraint solution is utilized as the initial condition to the nonlinear constraint routine. In all cases, the initial condition to the nonlinear algorithm was taken to be the $(2,2,1,1,1,1)$-solution, except in the hyperbolic $(L-S)$-case, in which a combination of the generic solution and the one provided by Lemma 5.2 ,

$$
(2,2,1,1,1,1)+(2,2,2,2,2,2)=(4,4,3,3,3,3),
$$

was chosen to make the algorithm converge. The numerical values of $\max (\delta / D)$ subject to the linear and nonlinear constraints for the various scalings and in the various spaces are as shown in Table 1 . The optimum edge lengths are shown in Table 2.

The $L, L+M+S$, and diam scalings behave in roughly the same way. As intuition suggests, one observes an increase of $\max _{\square} \delta(\square) / D(\square)$ from hyperbolic 


\begin{tabular}{c|c|c|c|c}
\hline $\sup _{A \xi \leq 0, c(\xi) \leq 0} \frac{\delta}{D}$ & $\begin{array}{c}\text { Hyperbolic } \\
\text { subject to } \\
\xi \geq 1 / \sqrt{-\kappa}\end{array}$ & $\begin{array}{c}\text { Hyperbolic } \\
\text { Euclidean } \\
\text { CAT }(0)\end{array}$ & Ptolemaic & Spherical \\
\hline$L$ & 0.1397 & 0.1464 & 0.1667 & 0.25 \\
$L+M+S$ & 0.0572 & 0.0607 & 0.0714 & 0.125 \\
$\operatorname{diam}$ & 0.2788 & 0.2929 & 0.2929 & 0.5 \\
$L-S$ & 0.5 & 0.5 & 0.5 & 0.5 \\
\hline
\end{tabular}

Table I. Achievable bounds in various spaces.

to spherical spaces. The hyperbolic $\xi \geq 1 / \sqrt{-\kappa}$ column indicates that for a hyperbolic quadrilateral with edge length bounded from below, $\sup (\delta / D)$ remains below what could be achieved without a lower bound, which, for infinitesimally small edge length, equals the Euclidean bound. On the other hand, there is a strict inequality between the absolute hyperbolic/Euclidean bound and the spherical bound. The Ptolemaic space appears "somewhere between" Euclidean and spherical spaces, which is not surprising, since $\mathbb{H}, \mathbb{E} \subseteq \mathbb{P}$.

The situation is totally different for the $L-S$ scaling, since $\max \delta /(L-S)$ remains constant across all spaces. For $\mathbb{H}, \mathbb{E}$, and $\mathbb{S}$, this seems to indicate that $\max \delta /(L-S)=0$ is indicative of constant curvature, but it is unclear what this means for $\mathbb{P}$.

Regarding the minima, no matter what the scaling is, we have $\min \delta / D=0$, since the latter is easily seen to be reached for the degenerate quadrilateral $a=c$, $b=d$, embeddable is all spaces considered.

\section{Geometric Interpretation of Suprema}

The numerical values of $\sup _{\square \subset \mathbb{H}, \mathbb{E}} \frac{\delta}{D}$ for $D=L, L+M+S$, diam indicate that the suprema are reached for a 2-dimensional square, $u=v, x=y, z=w$. Indeed, for $H$ with $\xi \geq 1$, we have $x=y=1$ and $z=w=1$, and some hyperbolic trigonometry in the right triangle $\triangle a b 0$, where $0=[a, d] \cap[b, c]$, yields

$$
u=v=2 \cosh ^{-1}(\sqrt{\cosh (1)})=1.3653,
$$

from which the result follows. For $\mathbb{E}$, the result is trivial to verify.

In positive curvature, the numerical results are consistent with a quadrilateral embedded in a 2-sphere, with the midsize and small diagonals $[a, b] \cup[b, d] \cup$ $[d, c] \cup[c, a]$ forming the "equator" and with the large diagonals $[a, d],[b, c]$ each 


\begin{tabular}{|l|c|c|c|c|}
\hline & $\begin{array}{c}\text { Hyperbolic } \\
\xi \geq 1 / \sqrt{-\kappa}\end{array}$ & $\begin{array}{c}\text { Euclidean } \\
\text { hyperbolic }\end{array}$ & Ptolemaic & Spherical \\
\hline \hline$L$ & 1.0000 & 1.2375 & 1.0608 & 3.1416 \\
& 0.9281 & 1.2375 & 0.5304 & 3.1416 \\
& 0.6948 & 0.8750 & 0.5304 & 1.5708 \\
& 0.6948 & 0.8750 & 0.5304 & 1.5708 \\
& 0.6949 & 0.8750 & 0.5304 & 1.5708 \\
& 0.6947 & 0.8750 & 0.5304 & 1.5708 \\
\hline$L+M+S$ & 1.0000 & 7.7272 & 1.0450 & 0.0496 \\
& 1.0000 & 7.7272 & 0.5225 & 0.0496 \\
& 0.6947 & 5.4640 & 0.5225 & 0.0246 \\
& 0.6945 & 5.4640 & 0.5225 & 0.0246 \\
& 0.6945 & 5.4640 & 0.5225 & 0.0246 \\
& 0.6944 & 5.4640 & 0.5225 & 0.0246 \\
\hline diam & 1.0000 & 7.6630 & 1.3077 & 0.0534 \\
& 1.0000 & 7.6630 & 1.3077 & 0.0534 \\
& 0.7212 & 5.4185 & 0.9247 & 0.0264 \\
& 0.7212 & 5.4185 & 0.9247 & 0.0264 \\
& 0.7212 & 5.4185 & 0.9247 & 0.0264 \\
& 0.7212 & 5.4185 & 0.9247 & 0.0264 \\
\hline$L-S$ & 3.7133 & 7.9753 & 8.2890 & 3.1416 \\
& 3.7133 & 7.9753 & 8.2890 & 3.1416 \\
& 3.0662 & 5.6407 & 5.8613 & 1.5708 \\
& 3.0662 & 5.6407 & 5.8613 & 1.5708 \\
& 3.0662 & 5.6407 & 5.8613 & 1.5708 \\
& 3.0662 & 5.6407 & 5.8613 & 1.5708 \\
\hline
\end{tabular}

Table 2. Optimum edge lengths in various spaces for various scalings.

half the circumference in length. On the unit sphere, this yields $x=y=u=v=$ $\frac{\pi}{2}$ and $u=v=\pi$, with which the numerical result is consistent.

\section{Proof of Bound for $\operatorname{CAT}(0)$ and Euclidean Spaces for Some Scalings}

Here, we endeavor to prove the bounds in the CAT(0) space for all scalings, except the diameter scaling, which entails additional expressions making the problem more involved. The Euclidean bound is proved for the $L$-scaling. The Euclidean bound in the $(L+M+S)$-scaling does not appear to be tractable with the technique presented here; it can, however, be proved by a Sturm-sequence argument implemented in Maple [Ariaei and Jonckheere 08]. 
As is well known, $\mathbb{E}, \mathbb{H} \subset \mathrm{CAT}(0)$, so that

$$
\sup _{\square \subset \mathbb{E}, \mathbb{H}} \frac{\delta}{D} \leq \sup _{\square \subset \mathrm{CAT}(0)} \frac{\delta}{D},
$$

and we endeavor to derive a bound on the right-hand side of the inequality and then show that this bound is achievable in $\mathbb{E}$.

The inequality (3.4) can be written as

$$
(u+v)^{2}-2 u v \leq(x+y)^{2}-2 x y+(z+w)^{2}-2 z w .
$$

Since the spaces $\mathbb{E}$ and $\mathbb{H}$ are known to be Ptolemaic, we can utilize the inequality

$$
u v \leq x y+z w
$$

in (7.1), which yields

$$
(u+v)^{2} \leq(x+y)^{2}+(z+w)^{2}, \quad L^{2} \leq M^{2}+S^{2} .
$$

Clearly, (3.4) implies (7.2), so that

$$
\sup _{u^{2}+v^{2} \leq x^{2}+y^{2}+z^{2}+w^{2}} \frac{\delta}{D} \leq \sup _{L^{2} \leq M^{2}+S^{2}} \frac{\delta}{D},
$$

and we proceed to derive an explicit bound on the right-hand side of this inequality and show that this bound in achievable in $\mathbb{E}$.

\section{I. $L$-Scaling, $\max \frac{\delta}{L}=\frac{(\sqrt{2}-1)}{2 \sqrt{2}}$}

To show that

$$
\max \frac{\delta}{L}=\frac{(\sqrt{2}-1)}{2 \sqrt{2}},
$$

it suffices to show the following:

$$
(u+v)-\sqrt{2}(x+y) \leq 0,
$$

or equivalently,

$$
L-\sqrt{2} M \leq 0 .
$$

Since $S \leq M$, from (7.2) we have

$$
L^{2} \leq 2 M^{2} .
$$

Therefore,

$$
L \leq \sqrt{2} M \Longrightarrow L-\sqrt{2} M \leq 0 .
$$


The proof is complete under the relaxed constraint $L^{2} \leq M^{2}+S^{2}$, and since the bound is obviously achieved for a flat Euclidean square, it is the bound for $\mathbb{E}$.

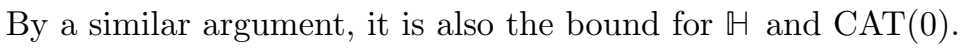

\section{2. $(L+M+S)$-Scaling, $\max \frac{\delta}{L+M+S}=\frac{\sqrt{2}-1}{2(\sqrt{2}+2)}$}

Verifying the bound "by hand" does not appear completely straightforward; however, it is easily manageable with the help of Mathematica. The latter indeed confirms that the following expression is true:

$$
\begin{gathered}
\forall(L, M, S):\left(L^{2} \leq M^{2}+S^{2}\right) \wedge(L \geq M \geq S \geq 0) \\
\Longrightarrow(L-M) \leq \frac{\sqrt{2}-1}{\sqrt{2}+2}(L+M+S) .
\end{gathered}
$$

\section{3. $(L-S)$-Scaling, $\max \frac{\delta}{L-S}=\frac{1}{2}$}

To prove that the inequality $\frac{\delta}{L-S} \leq \frac{1}{2}$ always holds, we use the assumption $L \geq$ $M \geq S$. Since $M \geq S$, the inequality $L-M \leq L-S$ holds. Therefore, $\frac{L-M}{2(L-S)} \leq$ $\frac{1}{2}$ is always true.

\section{Geometric Interpretation of Scaled FPC}

Here we show that the $L$-scaled 4 -point condition captures some "thinness" characteristics of the various geodesic triangles in the network. Consider a geodesic triangle $\Delta a b c$. It can be shown [Bridson and Haefliger 99, p. 408] that there exist quantities $r, s, t>0$ such that $d(a, b)=r+s, d(b, c)=s+t$, and $d(c, a)=t+r$. Define points $i_{a} \in[b, c], i_{b} \in[c, a]$, and $i_{c} \in[a, b]$ such that $d\left(b, i_{a}\right)=s, d\left(i_{a}, c\right)=t, d\left(c, i_{b}\right)=t, d\left(i_{b}, a\right)=r, d\left(a, i_{c}\right)=r$, and $d\left(i_{c}, b\right)=s$. The points $i_{a}, i_{b}$, and $i_{c}$ can be defined as the points of contact of the inscribed circle with sides $[b, c],[a, c]$, and $[a, b]$, respectively, of the comparison triangle. Consider the quadrilateral $\square a b i_{a} i_{b}$. It can be shown [Bridson and Haefliger 99, p. 411, Figure H.6] that $L=d\left(a, i_{a}\right)+d\left(b, i_{b}\right), M=d(a, b)+d\left(i_{b}, i_{a}\right)$. The $L$-scaled condition means that $1-\frac{M}{L} \leq\left(2 \bar{b}_{L}\right)$, where $\bar{b}_{L}$ is the bound in the space being analyzed; equivalently, $L\left(1-2 \bar{b}_{L}\right) \leq M$. For the quadrilateral $\square a b i_{a} i_{b}$, this yields

$$
\left(d\left(a, i_{a}\right)+d\left(b, i_{b}\right)\right)\left(1-2 \bar{b}_{L}\right) \leq r+s+d\left(i_{b}, i_{a}\right) \leq r+s+2 t .
$$


Applying the same reasoning to the other quadrilaterals and adding the resulting inequalities yields

$$
\left(d\left(a, i_{a}\right)+d\left(b, i_{b}\right)+d\left(c, i_{c}\right)\right) \leq \frac{1}{1-2 \bar{b}_{L}}(d(a, b)+d(b, c)+d(c, d)) .
$$

In other words, we obtain a bound on the sum of the distances between the vertices and the contact points between the opposite sides of the triangle and its inscribed circle as a function of the perimeter of the triangle. Clearly, this is a "fatness" bound.

Repeating the same argument for the $(L+M+S)$-scaling yields (the details are left to the reader)

$$
\left(d\left(a, i_{a}\right)+d\left(b, i_{b}\right)+d\left(c, i_{c}\right)\right) \leq \frac{1+3 \bar{b}_{L+M+S}}{1-2 \bar{b}_{L+M+S}}(d(a, b)+d(b, c)+d(c, d)) .
$$

\section{Simulation Experiments}

\section{I. Scaled FPC in Small-World, Scale-Free, and Other Graph Generators}

Parallel to what was done in [Jonckheere et al. 08], here we examine the behavior of $\delta(\square) / \operatorname{diam}(G)$, which is a large-scale approximation of $\delta(\square) / \operatorname{diam}(\square)$, for the traditional graph generators: the Erdős-Rényi purely random graphs, the Barabási-Albert growth/preferential attachment scale-free generator, and the Watts-Strogatz $\beta$-model small-world generator [Barabási et al. 99]. In addition, we also consider a slight variant of the growth/preferential attachment generator: that for which the attachment is uniform. Recall that the latter is not scale-free [Barabási et al. 99]. The scaling by the diameter of the graph rather than the diameter of the quadrilateral is motivated by the need to make the computation tractable.

To draw a fair comparison among all four models, we set the total number of nodes (50 in the experiment of Figure 2 and 100 in the experiment of Figure 3) and then adjust the parameters of the various models so as to have the same number of edges $M$ across all four models (for details, see [Jonckheere et al. 08] or [Lohsoonthorn 03], since the protocol of this FPC experiment is exactly the same as that of the TTC experiment of [Jonckheere et al. 08]). Then we plot

$$
E_{M}\left(\max _{\square \subset G} \frac{\delta(\square)}{\operatorname{diam}(G)}\right)
$$

versus $M$, where $E_{M}$ denotes the ensemble average over all graphs of size $M$ generated by all four models. The results are shown in Figures 2 and 3 . 


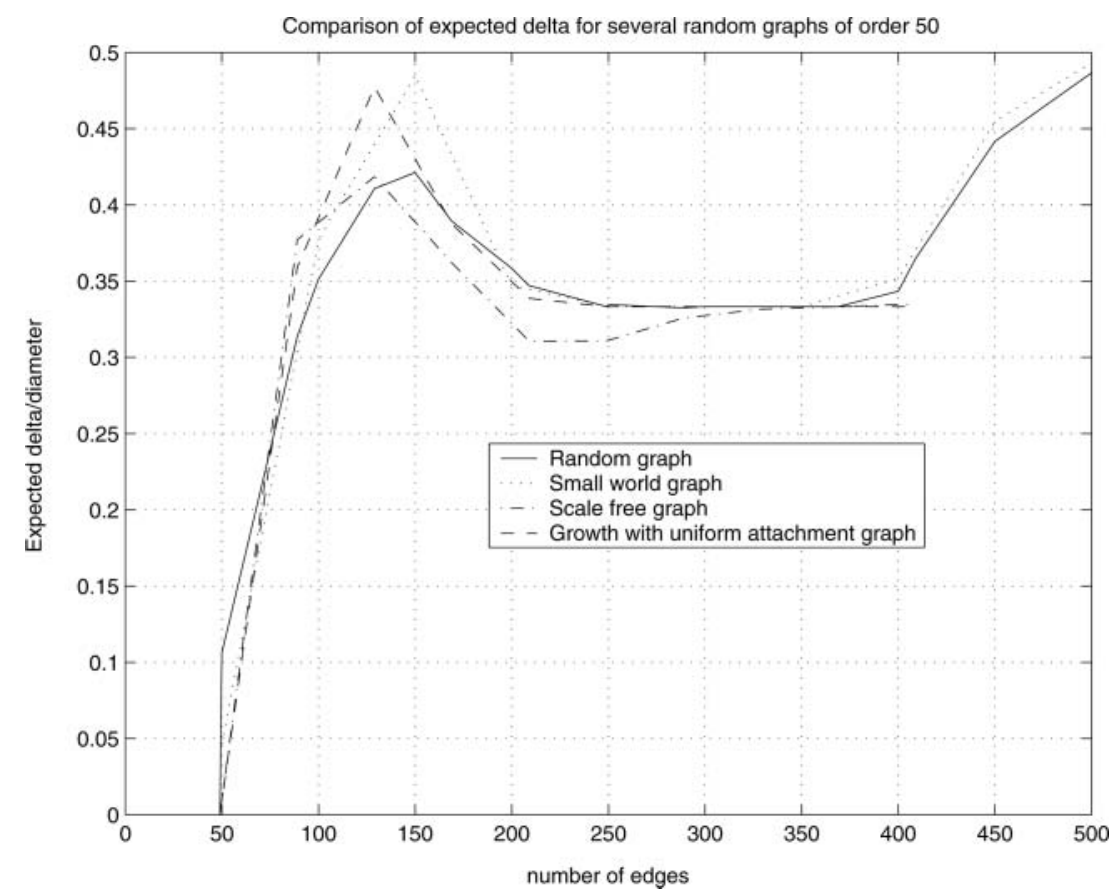

Figure 2. The mathematical expectation of $\max (\delta(\square) / \operatorname{diam}(G))$ versus the total number of edges $M$ for all four graph generators of order 50 . Observe that the scale-free graph is the closest to being hyperbolic.

The results are consistent with those of the scaled TTC [Jonckheere et al. 08], in the sense that among all graph generators, the Barabási-Albert scale-free one comes closest to being scaled Gromov hyperbolic. More specifically, this phenomenon happens in an intermediate range of values of $M$, not too small, for otherwise, the graph looks like the startup tree $\left(\delta_{\mathrm{FPC}}(\square)=0\right)$, and not too large, for otherwise, the graph has too many quadrilaterals with the potential for too-high values of $\delta_{\mathrm{FPC}}$. In this region, though, there is a mild discrepancy with [Jonckheere et al. 08], in the sense that the FPC performance (9.1) does not quite drop below the theoretical threshold as the TTC performance did.

This discrepancy can be explained on the ground that the graphs on which the TTC and FPC were tested involve some randomness in the definition of the startup backbone and the attachment process, so that in the FPC experiment it was nearly impossible to reproduce the graphs of the TTC experiment. Moreover, the performance is evaluated in a very conservative way, since for every graph the worst quadrilateral (with the highest $\delta_{\mathrm{FPC}}(\square) / \operatorname{diam}(\square)$ ) is chosen, making 


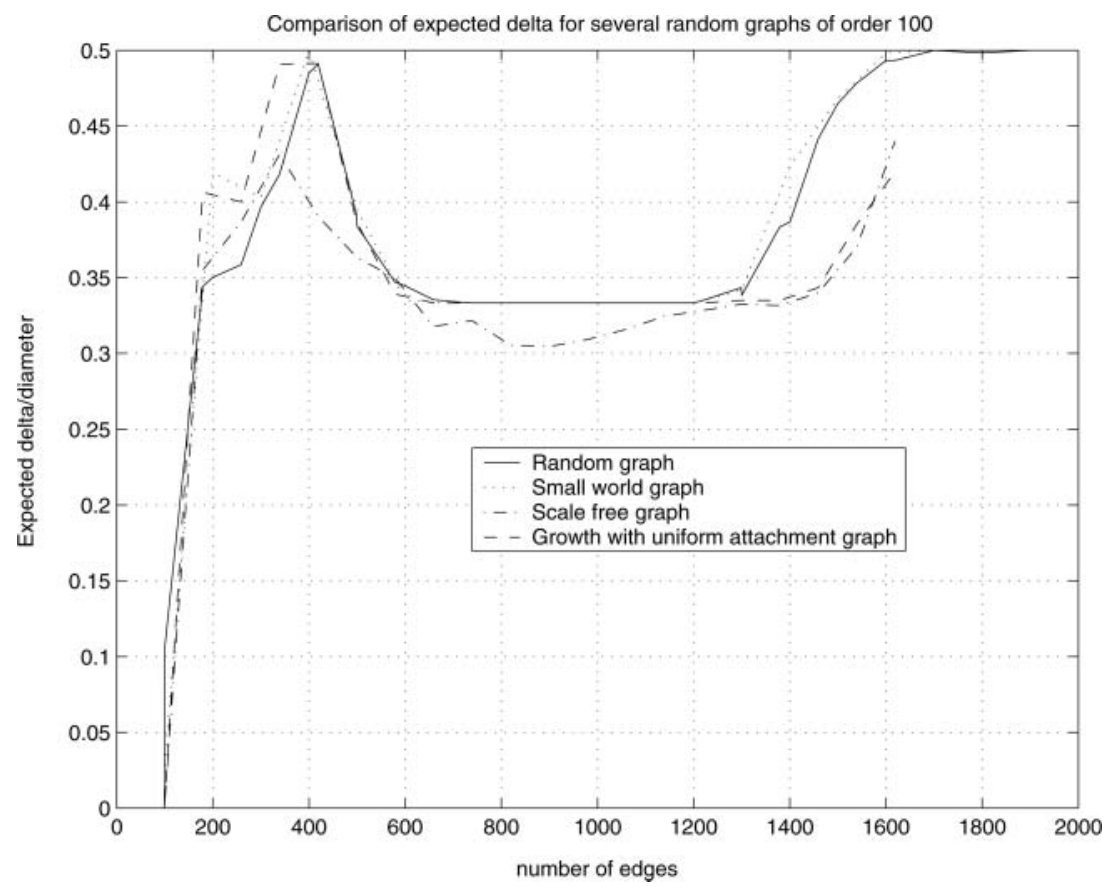

Figure 3. The mathematical expectation of $\max (\delta(\square) / \operatorname{diam}(G))$ versus the total number of edges $M$ for all four graph generators of order 100. Observe that the scale-free graph is the closest to being hyperbolic.

the performance sensitive to random events in the backbone and the attachment process.

The findings of Figures 2 and 3 are consistent with the "taxonomy of large-scale networks" of [Narayan and Saniee 09, Figure 5], showing that the relationships among the various network concepts (power law, scale-free, hyperbolic, etc.) are not inclusions, but rather nonempty intersections; e.g., there are power law graphs that are hyperbolic, while other power law graphs are not hyperbolic. To further exemplify the fact that scale-free graphs need not be hyperbolic, it was observed in [Boguna et al. 10] that scale-free networks do not show the trafficcongestion anomalies reported in [Jonckheere et al. 11b, Lou et al. 11, Narayan and Saniee 09] for hyperbolic networks.

Graphs of order 100, such as those utilized in the simulation studies, might appear small by today's standards; however, the scaled $\delta_{\mathrm{FPC}}$ analysis on graphs of order 500 was done in a recent experiment on spin networks [Jonckheere et al. 11a]. In any case, the next experiment will involve many more vertices. 


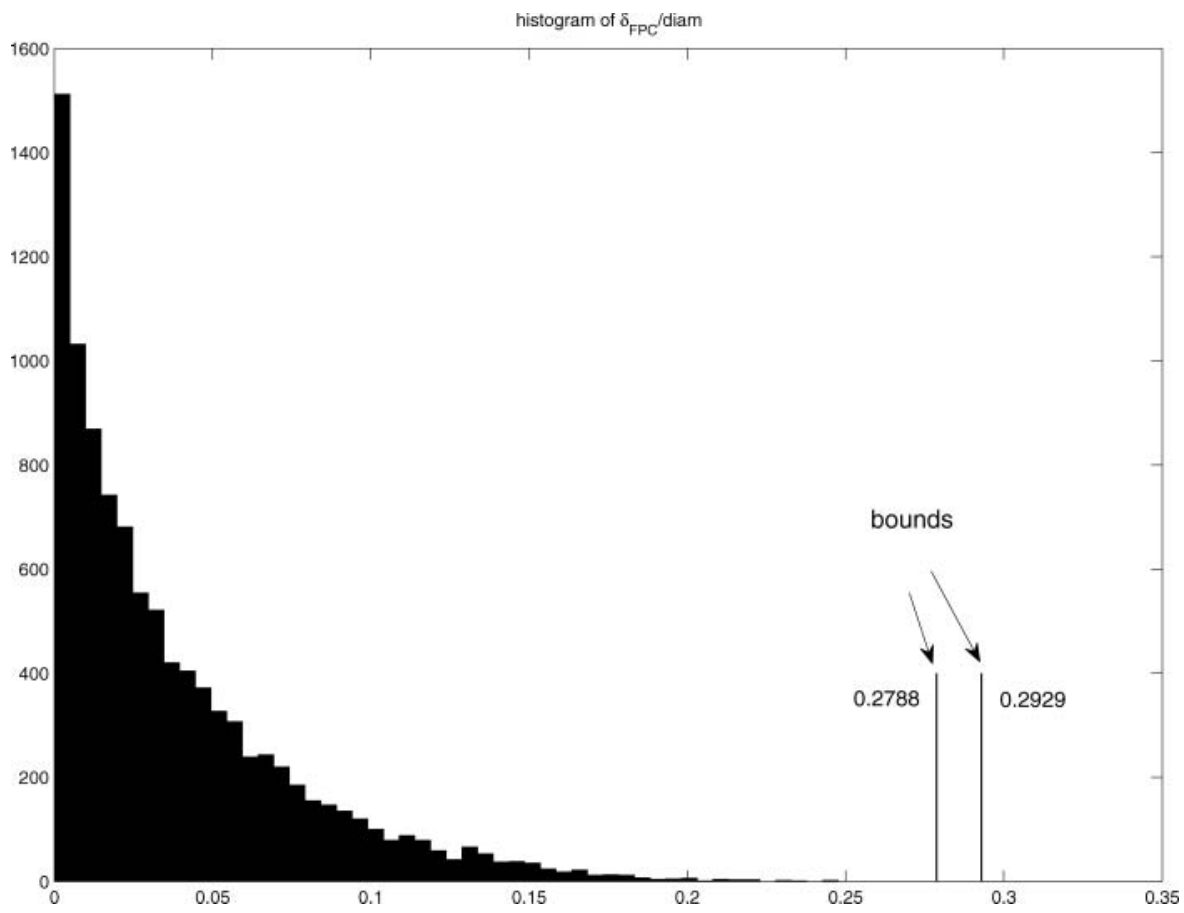

Figure 4. Histogram of $\delta_{\mathrm{FPC}}(\square) / \operatorname{diam}(\square)$ for 40,000 points uniformly chosen over the open Poincaré disk.

\subsection{Poincare Disk Network}

To see how the scaled FPC test behaves for a truly hyperbolic network, take the Poincaré disk; uniformly sample the open unit disk of the complex plane (the norm is uniformly distributed over $[0,1)$ and the argument is uniformly distributed in $[0,2 \pi)$ ). Let us pick 40,000 points and let us plot the histograms of $\delta_{\mathrm{FPC}}(\square) / \operatorname{diam}(\square)$. It can be seen in Figure 4 that the theoretically established bounds are never exceeded.

\section{Conclusions}

We have shown that scaling the Gromov four-point condition in various ways and requiring the various scaled quantities to be below the corresponding hyperbolic threshold leads to a concept of Gromov hyperbolic space applicable to finite spaces, revealing a new "thinness" property of triangles, and relevant 
to the classical network graph generators. But probably the deepest question raised here is what kind of spaces "fill" the discontinuity of $\sup _{\square} \delta(\square) / D(\square)$ between Euclidean spaces and Riemannian manifolds of constant positive curvature. These spaces seem to defy constant-curvature Riemannian geometry. The Ptolemaic spaces appear to be such spaces, but whether other such spaces can be identified is widely open. Another widely open application-oriented question is whether there are graphs that can be modeled as Ptolemaic spaces.

\section{Appendix: Computational Algebra for the Ptolemaic Case}

If $D(\xi)$ is a polynomial in $\xi=(u, v, x, y, z, w)$, we write

$$
\sup _{\xi, f(\xi)<0} \frac{\delta(\xi)}{D(\xi)}=\bar{b},
$$

where $f(\xi)=\left(c(\xi) \xi^{\prime} A^{\prime}\right)^{\prime}$, where $c(\xi)<0$ are the polynomial embeddability constraints in either Euclidean or Ptolemaic space and $A \xi<0$ are the linear constraints. The above can then be rewritten, in polynomial format, as follows:

$$
f_{0}(\xi):=u+v-(x+y)-2 \bar{b} D(u, v, x, y, z, w)<0 .
$$

If the scaling factor $D$ is the largest sum of lengths of diagonals, $L$, the above becomes

$$
f_{0}(\xi):=u+v-(x+y)-2 \bar{b}(u+v)<0 .
$$

If $\delta$ is scaled by the perimeter of the quadrilateral, then

$$
f_{0}(\xi):=u+v-(x+y)-2 \bar{b}(u+v+x+y+z+w)<0 .
$$

In the Euclidean case and for the scaling $D=L$, we have a good guess as to what $\bar{b}$ is, namely $\frac{\sqrt{2}-1}{2 \sqrt{2}}$. It is even an algebraic number, so that the problem can be reduced to one over $\mathbb{Z}[\xi]$, but for the sake of the simplicity of the exposition, we will not pursue this here.

In the Euclidean case with $D=L$, the statement (11.1) can be rephrased algebraically as the conjunction $P \wedge \neg Q$ of two statements: The first one, $P$, asserts that for all $\xi$ satisfying the constraints, the scaled $\delta$ remains below the bound; the second one, $\neg Q$, asserts that there does not exist any $\xi$ satisfying the constraints and giving a scaled $\delta$ above the bound.

Formally, the first statement is to decide whether it is true that whenever $f_{1}(\xi)<0, \ldots, f_{M}(\xi)<0$, we have $f_{0}(\xi)<0$. In the predicate language $L_{1}$ (see [Manin 77]), this statement is written as the formula

$$
P:=(\forall \xi)\left(\left(f_{1}(\xi)<0 \wedge \cdots \wedge f_{M}(\xi)<0\right) \rightarrow f_{0}(\xi)<0\right)
$$


being true. In the Mathematica language, the above formula is written as

$$
\text { ForALL }\left[\left\{\xi_{1}, \ldots, \xi_{M}\right\}, \quad f_{1}<0 \& \& \cdots \& \& f_{N}<0, f_{0}<0\right] .
$$

In Mathematica, it might take several instructions of the form

$$
\text { FullSimplify }[\%]
$$

before a true or false answer is rendered. The above is, formally, the universal quantifier $\forall$ elimination.

The second statement can be formally reworded as the nonexistence of real solutions to the system of polynomial equations $f_{1}<0, \ldots, f_{M}<0$ and $f_{0}>0$. In the $L_{1}$ language, we have to decide whether the formula

$$
Q:=(\exists \xi)\left(f_{1}<0 \wedge \cdots \wedge f_{M}<0 \wedge f_{0}>0\right)
$$

is false. In Mathematica, the above formula is written

$$
\text { Exists }\left[\left\{\xi_{1}, \ldots, \xi_{N}\right\}, f_{1}<0 \& \& \cdots \& \& f_{M}<0 \& \& f_{0}>0\right]
$$

Again, it might take several iterations on

$$
\text { Resolve [\%] }
$$

before a final true or false answer is rendered. Formally, the above is elimination of the existential quantifier $\exists$.

Thus what needs to be established is that $P \wedge \neg Q$ is true.

Even in the simplified Ptolemaic case, with all constraints properly taken into consideration, the quantifier elimination ForAll seems to be running forever (a run of at least 12 hours has been observed!). It appears, therefore, that we ought to simplify the problem "by hand" before submitting it to Mathematica.

\section{II.I. Tarski-Seidenberg Decision for the Ptolemaic Case, $L$-Scaling}

In $L=u+v$ scaling, what is overlooked if we just submit the problem "as is" to Mathematica is the independence of the criterion

$$
(u+v)-(x+y) \leq 2 \bar{b}(u+v)
$$

of the $z, w$ variables. Therefore, those variables are candidates for elimination "by hand."

More formally, let $\mathcal{B}(u, v, x, y, z, w)$ be the Boolean combination of polynomial inequality constraints of the problem, that is, the sign constraints, the triangle inequalities, the convention on opposite diagonals, and the Ptolemaic conditions. Elimination of $z, w$ consists in deriving a Boolean combination $\mathcal{B}(u, v, x, y)$ such that there exists $(z, w)$ such that $\mathcal{B}(u, v, x, y, z, w)$ is true if and only if $\mathcal{B}(u, v, x, y)$ is true. 
II.I.I. Convention and Sign Constraints. Recall that $(u, v)$ and $(x, y)$ are pairs of lengths of diagonals such that $u+v \geq x+y \geq z+w$. This leaves us the freedom to order the pairs $(u, v)$ and $(x, y)$ as follows, where we have by the same token enforced the fact that lengths are nonnegative:

$$
x \geq y \geq 0, \quad v \geq u \geq 0 .
$$

The reasons for this particular ordering will become clearer later.

II.I.2. Triangle Inequalities. Working out the various triangle inequalities, we obtain the following relevant inequalities (those that are trivial have been omitted):

$$
\begin{aligned}
& y+v \geq w \geq u-x, \quad x+v \geq z \geq u-y, \\
& x+u \geq w \geq v-y, \quad y+u \geq z \geq v-x .
\end{aligned}
$$

Using the convention on the ordering of the pairs $(u, v)$ and $(x, y)$, the above reduces to

$$
\begin{aligned}
y+v & \geq w, \\
z & \geq u-y, \\
x+u & \geq w \geq v-y, \\
y+u & \geq z \geq v-x .
\end{aligned}
$$

Clearly, necessary conditions for existence of $(z, w)$ include the inequalities between the leftmost and rightmost terms of the bottom two strings. The latter is easily seen to reduce to

$$
v \leq x+u+y
$$

Observe that this resulting inequality is no longer a "triangle" inequality, since after removing the opposite diagonals $[a, c]$ of length $z$ and $[b, d]$ of length $w$, the resulting quadrilateral is no longer complete; it has no triangles. In fact, the above is a polygonal inequality.

II.I.3. Opposite Diagonal Conditions. The opposite diagonal conditions are

$$
u+v \geq x+y \geq z+w
$$

Using the above triangle inequalities to bound $z, w$ from above, we obtain the following:

$$
\begin{aligned}
u+v & \geq x+y \geq z+w \geq(u+v)-2 y \geq(u+v)-2 x \geq 2 u-(x+y) \\
& \geq 2 v-(x+y) .
\end{aligned}
$$


Using the convention on the ordering of $u, v, x, y$, the above simplifies to

$$
u+v \geq x+y \geq z+w \geq(u+v)-2 y \geq 2 v-(x+y) .
$$

Therefore, necessary conditions to be able to eliminate $z, w$ are the inequalities between the two leftmost and rightmost terms, which yield

$$
\begin{aligned}
& u+v \geq x+y \geq(u+v)-2 y \\
& x+y \geq v
\end{aligned}
$$

II.I.4. Ptolemaic Conditions. The Ptolemaic conditions are trivially rewritten

$$
u v-x y, x y-u v \leq z w \leq u v+x y .
$$

The inequality between the two extreme terms of (11.11) is trivial. However, the first inequality and the various triangle inequalities in the triangles having $z, w$ as sides yield

$$
\begin{aligned}
|u v-x y| & \leq z w \leq(y+u)(y+v) \leq(x+v)(y+v) \leq(x+v)(x+u) \\
& \leq(y+u)(x+u)
\end{aligned}
$$

Utilizing $x \geq y, v \geq u \Rightarrow(x+v) \geq(u+y)$, the above simplifies to

$$
|u v-x y| \leq z w \leq(y+u)(y+v) \leq(y+u)(x+u) .
$$

Again, the inequalities between the extreme terms eliminate $z, w$. Clearly, there are four such inequalities. Two of them are easily found to be trivial, while the nontrivial ones are

$$
u v \leq 2 x y+u^{2}+u x+u y, \quad x y \leq 2 u v+y^{2}+u y+v y .
$$

Finally, returning to the second inequality of (11.11) and using triangle inequalities in those triangles having $z, w$ as sides yields

$$
\begin{aligned}
& (u-y)(v-y) \leq z w \leq u v+x y, \quad(u-y)(u-x) \leq z w \leq u v+x y \\
& (v-x)(v-y) \leq z w \leq u v+x y, \quad(v-x)(u-x) \leq z w \leq u v+x y
\end{aligned}
$$

Expanding and simplifying yields the (linear!) constraints

$$
y-(u+v) \leq x, \quad u-(x+y) \leq v, \quad v-(x+y) \leq u, \quad x-(u+v) \leq y .
$$

Finally, using the convention $x \geq y, v \geq u$, we get

$$
y-x \leq u+v, \quad v-u \leq x+y .
$$

Observe that the above are polygonal inequalities in the incomplete quadrilateral. 
II.I.5. Necessary Conditions. Necessary conditions for the existence of $z, w$ such that $\mathcal{B}(u, v, x, y, z, w)$ is true can be expressed as follows:

$$
\begin{aligned}
\mathcal{B}(u, v, x, y) & \subseteq \\
\subseteq & (x \geq y \geq 0) \wedge(v \geq u \geq 0) \wedge(v \leq x+u+y) \\
& \wedge(u+v \geq x+y \geq u+v-2 y) \wedge(x+y \geq v) \\
& \wedge\left(u v \leq 2 x y+u^{2}+u x+u y\right) \wedge\left(x y \leq 2 u v+y^{2}+u y+v y\right) \\
& \wedge(y-x \leq u+v) \wedge(v-u \leq x+y) .
\end{aligned}
$$

II.I.6. Sufficient Conditions. In the preceding, we have written estimates of the form $\underline{z} \leq z \leq \bar{z}, \underline{w} \leq w \leq \bar{w}, \underline{s} \leq z+w \leq \bar{s}$, and $\underline{p} \leq z w \leq \bar{p}$, and we have written $\underline{z}(u, v, x, y) \leq \bar{z}(u, v, x, y)$, etc., as necessary conditions for the existence of, and hence the possibility of eliminating, $(z, w)$. These conditions are obviously not sufficient, since clearly a discriminant condition is needed. The latter is the inherently difficult step in this computer algebra problem. Indeed, attempting to execute the apparently simple Mathematica instructions

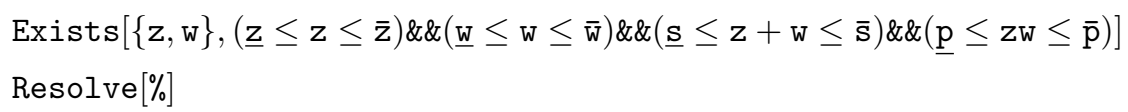

results in Mathematica running endlessly. The reason is that while the above is simple to express geometrically in the $(z, w)$-plane, it is linguistically difficult to express in the $L_{1}$ language.

Geometrically, we have to secure a nonempty intersection between a rectangle implementing the triangle inequalities (11.2)-(11.5), the region between two hyperbolas implementing the Ptolemaic conditions (11.11), and a half-plane with boundary line at a $-45^{\circ}$ angle implementing the opposite diagonal condition, the second inequality of (11.7).

There are two cases to be considered: $y+v>x+u$ and $y+v<x+u$. If $y+$ $v>x+u$, the rectangle is $[v-x, y+u] \times[v-y, x+u]$, the "large" rectangle of Figure 5; if $y+v<x+u$, the rectangle is $[u-y, y+u] \times[v-y, v+y]$, the "small" rectangle of Figure 6. In either case, the hyperbolas are $z w= \pm(u v-x y)$ and $z w=u v+x y$. The $-45^{\circ}$ boundary line of the half-plane is $z+w=x+y$. We have also drawn the lines $z+w=u+v-2 y$ and $z+w=2 v-(x+y)$ that saturate the inequalities (11.8), although this is not absolutely necessary.

Take the "small" rectangle $[u-y, y+u] \times[v-y, v+y]$. Clearly, the line $z+$ $w=x+y$ has to be above the point $(u-y, v-y)$, which requires $u+v<x+$ $3 y$. Next, we look at the position of the hyperbola $z w=|x y-u v|$ relative to the point $(u-y, v-y)$. It is easily seen that as a corollary of $u+v>x+y$, we have 


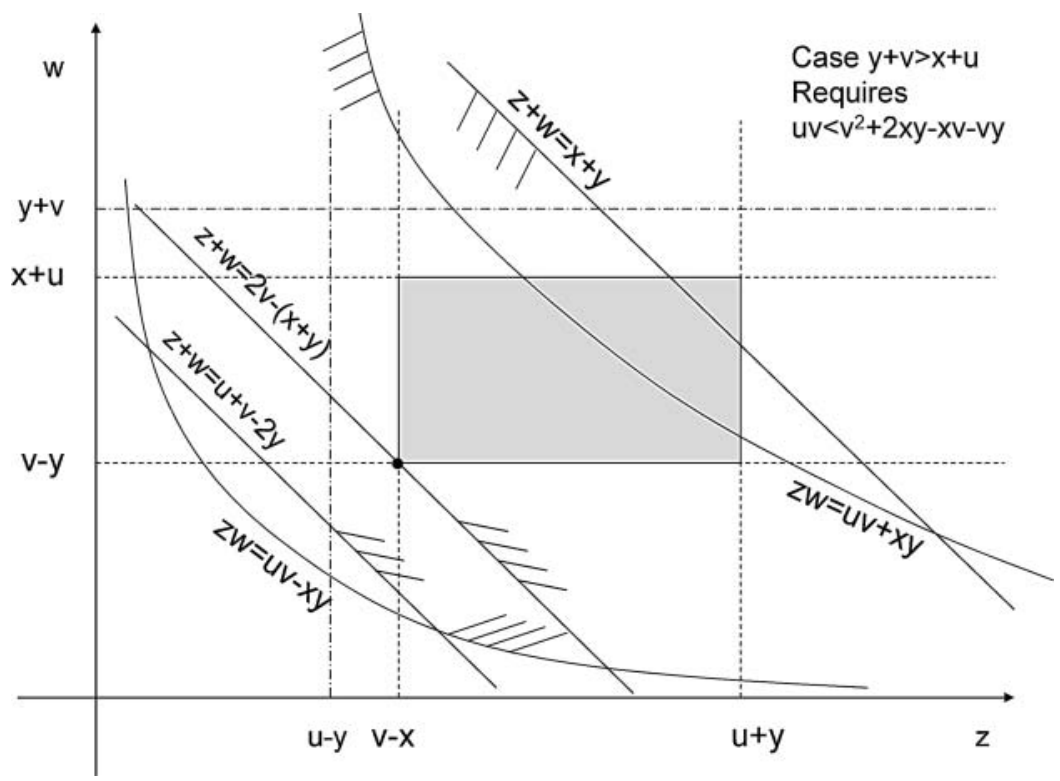

Figure 5. Illustration of the discriminant constraints in the $(z, w)$-plane for the case of the "large rectangle" $y+v>x+u$.

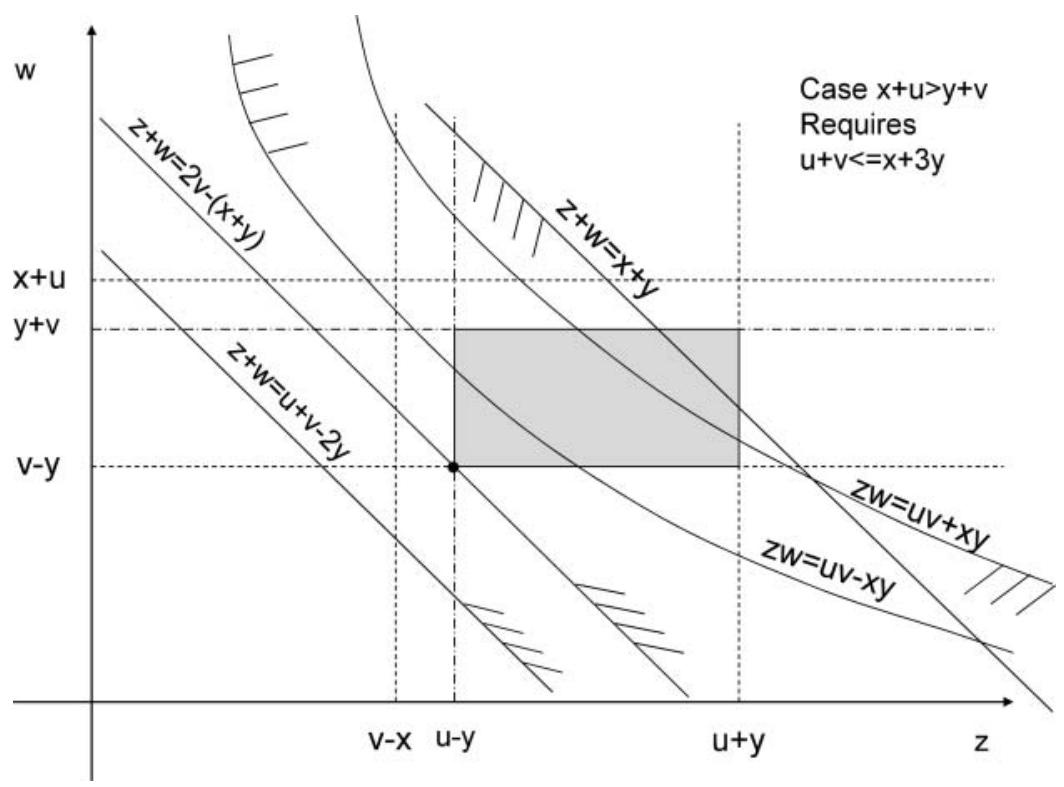

Figure 6. Illustration of discriminant constraints in the $(z, w)$-plane for the case of the "small rectangle" $y+v<x+u$. 
$u v-x y>(v-y)(u-y)$, so that the hyperbola $z w=u v-x y$ has to be "above" the point $(u-y, v-y)$. Clearly, the hyperbola $z w=x y-u v$ is irrelevant.

Because of the Ptolemaic conditions (11.11), the hyperbola $z w=x y+u v$ is "above" the hyperbola $z w=u v-x y$. Therefore, the discriminant issue is the position of the line $z+w=x+y$ relative to the hyperbola $z w=u v-x y$. The line and the hyperbola must intersect, which requires the classical condition

$$
(x+y)^{2}-4(u v-x y) \geq 0 .
$$

The difficulty is to state - linguistically - that the "crescent" between the line and the hyperbola intersects the rectangle. Therein lies the problem.

Regarding the "big rectangle" case, first of all, the line $z+w=x+y$ has to be "above" the point $(v-x, v-y)$, that is, $x+y>v-x+v-y$, which trivially holds in view of the opposite diagonal condition (11.10). The hyperbola $z w=$ $x y-u v$ is below the point $(v-x, v-y)$, that is, $x y-u v<(v-x)(v-y)$, as is easily seen from the opposite diagonal condition. Therefore, the hyperbola $z w=x y-u v$ is irrelevant. The hyperbola $z w=u v-x y$ could be either below or above the point $(v-x, v-y)$, depending on whether $u v<v^{2}+2 x y-y v-v x$ or $u v>v^{2}+2 x y-y v-v x$. The latter case contradicts the condition of the "big rectangle" case, so that the former prevails.

Hence we restrict ourselves to the situation $u v<v^{2}+2 x y-y v-v x$, in which the hyperbola $z w=u v-x y$ is irrelevant. In this case, all that remains to be imposed is that the hyperbola $z w=u v+x y$ be above the point $(v-x, v-y)$, that is, $u v+x y>(v-y)(v-x)$, which reduces to the polygonal inequality $v<$ $u+x+y$, already singled out in (11.6).

\section{II.2. Mathematica Encoding and Results}

In this subsection, we specifically write down the Mathematica instructions that implement the preceding ideas. The critical parameter of course is $\bar{b}$. We did "trial and error" for several different values of $\bar{b}$. This affects only the "c" (cost) expression. The other expressions implementing the Ptolemaic conditions, the triangle inequalities, etc., remain the same.

II.2.I. Triangle Inequalities. In Mathematica, the triangle inequalities are written

$$
\mathrm{t}=(\mathrm{v}<\mathrm{x}+\mathrm{u}+\mathrm{y}) .
$$

II.2.2. Sign and Opposite Diagonal Constraints. The sign and opposite diagonal constraints are written as

$$
\begin{aligned}
& \mathrm{s}=(\mathrm{x}>\mathrm{y}>0) \& \&(\mathrm{v}>\mathrm{u}>0) \\
& \mathrm{d}=(\mathrm{u}+\mathrm{v}>\mathrm{x}+\mathrm{y}) \& \&(\mathrm{x}+\mathrm{y}>\mathrm{u}+\mathrm{v}-2 \mathrm{y}) \& \&(\mathrm{x}+\mathrm{y}>\mathrm{v})
\end{aligned}
$$


1I.2.3. Ptolemaic Conditions. The Ptolemaic conditions split into two sets of constraints: the nonlinear p-constraints and the linear q-constraints. There are, respectively, as follows:

$$
\begin{aligned}
& \mathrm{p}=\left(\mathrm{uv}<2 \mathrm{xy}+\mathrm{u}^{\wedge} 2+\mathrm{ux}+\mathrm{u} \mathrm{y}\right) \& \&\left(\mathrm{xy}<2 \mathrm{uv}+\mathrm{y}^{\wedge} 2+\mathrm{uy}+\mathrm{vy}\right) \\
& \mathrm{q}=(\mathrm{v}-\mathrm{u}<\mathrm{x}+\mathrm{y}) \& \&(\mathrm{y}-\mathrm{x}<\mathrm{u}+\mathrm{v}) .
\end{aligned}
$$

11.2.4. Cost Criterion and Results. Computational results give $\bar{b} \approx 0.1667$, hence $2 \bar{b} \approx$ 0.3334. In fact, the numerical value of $(u, v, x, y, z, w)$ indicates that the optimum is obtained for a quadrilateral that degenerates along a line, so that it is fair to conjecture that $2 \bar{b}=1 / 3$. The problem is that Mathematica has some difficulties in handling the discriminant conditions. So we start with the necessary conditions and ascertain what bound is reached, so as to get an idea as to whether the discriminant conditions saturate.

11.2.5. Necessary Conditions: $p \wedge q \wedge s \wedge t \wedge d$. The cost is encoded as

$$
\mathrm{c}=(\mathrm{u}+\mathrm{v}-\mathrm{x}-\mathrm{y}<(\mathrm{u}+\mathrm{v}) / 3)
$$

Then we submit the following query to Mathematica:

$$
\text { ForAll }[\{\mathrm{u}, \mathrm{v}, \mathrm{x}, \mathrm{y}\}, \mathrm{p} \& \& q \& \& s \& \& t \& \& d, \mathrm{c}] \text {. }
$$

Immediately thereafter, Mathematica merely rewrites the constraints and the cost criterion. Then we ask Mathematica to simplify the expression

$$
\text { FullSimplify }[\%] \text {. }
$$

After about a minute, Mathematica renders the verdict False. This clearly indicates that the discriminant conditions play a role.

For the sake of argument, we attempt to establish the bound, disregarding the discriminant conditions. For each of the tentative values of $2 \bar{b}$ in the following table, Mathematica provides an answer within one minute:

\begin{tabular}{r|cccccc}
$2 \bar{b}$ & $1 / 3$ & $5 / 12$ & $11 / 24$ & $23 / 48$ & $47 / 96$ & $1 / 2$ \\
\hline True/False & F & F & F & F & F & T
\end{tabular}

It follows from the above that the bound, disregarding the discriminant conditions, appears to be $1 / 2$. To confirm this conjecture, we check $\exists(u, v, z, w)$ : $p \wedge q \wedge s \wedge t \wedge d \wedge(\neg c)$. In Mathematica,

$$
\begin{aligned}
& \text { Exists }[\{\mathrm{u}, \mathrm{v}, \mathrm{x}, \mathrm{y}\}, \mathrm{p} \& \& q \& \mathrm{q} \& \& \mathrm{t} \& \& \mathrm{~d} \& \&(! \mathrm{c})] \\
& \text { Resolve[\%] }
\end{aligned}
$$

Mathematica returns the answer False. Hence the bound $2 \bar{b}=1 / 2$ disregarding the discriminant conditions is confirmed. 
II.2.6. Necessary and Sufficient Conditions. We first look at the "small rectangle" case. The situation of Figure 6 is linguistically rect $\wedge r$, where

$$
\text { rect }=(u+x>v+y) \wedge(u+v<x+3 y), \quad r=\left((x+y)^{2}-4(u v-x y)>0\right) \text {. }
$$

In Mathematica,

$$
\begin{aligned}
& \text { rect }=(u+x>v+y) \& \&(u+v<x+3 y), \\
& r=\left((x+y)^{2}-4(u v-x y)>0\right) .
\end{aligned}
$$

Temporarily disregarding the condition of the nonempty intersection of the rectangle and the crescent, we eliminate $(z, w)$ by the following query:

$$
\forall(u, v, x, y):(p \wedge q \wedge s \wedge t \wedge d \wedge \operatorname{rect} \wedge r) \Rightarrow c,
$$

where $c$ is computed with the guessed bound $1 / 3$. In Mathematica,

$$
\begin{aligned}
& \text { ForAll }[\{u, v, x, y\}, p \& \& q \& \& s \& \& t \& \& d \& \& r e c t \& \& r, c] \\
& \text { FullSimplify }[\%]
\end{aligned}
$$

After a few minutes, Mathematica returns the answer True, as expected.

We now look at the "big rectangle" case. In this case, the only additional condition relative to the necessary conditions is

$$
\text { rect }=(y+v>x+u) \wedge\left(u v<v^{2}+2 x y-x v-v y\right) .
$$

In Mathematica,

$$
\text { rect }=(y+v>x+u) \& \&\left(u v<v^{2}+2 x y-x v-v y\right) .
$$

Hence we have to check whether

$$
\forall(u, v, x, y):(p \wedge q \wedge s \wedge t \wedge d \wedge \text { rect }) \Rightarrow c,
$$

where $c$ is computed with the guessed bound $1 / 3$. In Mathematica,

$$
\begin{aligned}
& \text { ForAll }[\{\mathrm{u}, \mathrm{v}, \mathrm{x}, \mathrm{y}\}, \text { p\&\&q\&\&s\&\&t\&\&d\&\&rect,} \mathrm{c}] \\
& \text { FullSimplify }[\%]
\end{aligned}
$$

After about five minutes, Mathematica returns the verdict True, as expected.

The conclusion is that adding the discriminant condition to the necessary condition case makes the upper bound drop from $1 / 2$ to $1 / 3$, as can reasonably be expected.

Acknowledgments. This research was supported by NSF Grant NetSE 1017881 and DARPA Contract N66001-00-C-8044. 


\section{References}

[Appa 02] G. Appa. "On the Uniqueness of Solutions to Linear Programs." Journal of the Operational Research Society 53 (2002), 1127-1132.

[Ariaei and Jonckheere 08] F. Ariaei and E. Jonckheere. "Upper Bound on Scaled Gromov 4-Point Delta: Sturm Sequence Approach." Available online (http://eudoxus. usc.edu/IW/AIA.html), 2008.

[Barabási et al. 99] Albert-László Barabási, Réka Albert, and Hawoong Jeong. "MeanField Theory for Scale-Free Random Networks." Physica A 272 (1999), 173-187.

[Berg and Nikolaev 08] I. D. Berg and I. G. Nikolaev. "Quasilinearization and Curvature of Alexandrov Spaces." Geom. Dedicata 133 (2008), 195-218.

[Blumenthal 53] L. M. Blumenthal. Theory and Applications of Distance Geometry. London: Oxford University Press, 1953.

[Boguna et al. 10] M. Boguna, F. Papadopoulos, and D. Krioukov. "Sustaining the Internet with Hyperbolic Mapping." Nature Communications 1 (2010), 62.

[Bridson and Haefliger 99] Martin R. Bridson and André Haefliger. Metric Spaces of Non-positive Curvature, A Series of Comprehensive Surveys in Mathematics 319. New York: Springer, 1999.

[Jonckheere et al. 07] Edmond A. Jonckheere, Mingji Lou, Prabir Barooah, and João P. Hespanha. "Effective Resistance of Gromov-Hyperbolic Graphs: Application to Asymptotic Sensor Network Problems." In IEEE Conference on Decision and Control, New Orleans, December 2007, pp. 1453-1458.

[Jonckheere et al. 08] E. Jonckheere, P. Lohsoonthorn, and F. Bonahon. "Scaled Gromov Hyperbolic Graphs." Journal of Graph Theory 57 (2008), 157-180.

[Jonckheere et al. 11a] E. Jonckheere, S. Schirmer, and F. Langbein. "Geometry and Curvature of Spin Network." To appear in 2011 IEEE Multi-Conference on Systems and Control, Denver, CO, September 2011. Available at arXiv:1102.3208v1 [quant-ph].

[Jonckheere et al. 11b] Edmond Jonckheere, Mingji Lou, Francis Bonahon, and Yuliy Baryshnikov. "Euclidean versus Hyperbolic Congestion in Idealized versus Experimental Networks." Internet Mathematics 7:1 (2011), 1-27.

[Jost 97] J. Jost. Nonpositive Curvature: Geometric and Analytic Aspects. Basel: Birkhäuser, 1997.

[Lohsoonthorn 03] P. Lohsoonthorn. "Hyperbolic Geometry of Networks." PhD thesis, Department of Electrical Engineering-Systems, University of Southern California, 2003. Available online (http://eudoxus.usc.edu/IW/MATTFINALTHESIS_MAIN. pdf).

[Lou et al. 11] M. Lou, E. Jonckheere, Y. Baryshnikov, F. Bonahon, and B. Krishnamachari. "Load Balancing by Network Curvature Control." International Journal of Computers, Communications and Control (IJCCC) 6:1 (2011), 134-149.

[Luenberger 89] D. G. Luenberger. Linear and Nonlinear Programming. Boston: Addison-Wesley, 1989. 
[Manin 77] Yu. I. Manin. A Course in Mathematical Logic. New York: Springer, 1977.

[Murakami and Ushijima 05] J. Murakami and A. Ushijima. "A Volume Formula for Hyperbolic Tetrahedra in Terms of Edge Lengths." Journal of Geometry 83:1-2 (2005), 153-163.

[Narayan and Saniee 09] O. Narayan and I. Saniee. "The Large Scale Curvature of Networks." arXiv:0907.1478v1, [cond-mat.stat-mech], 2009.

[Su 04] Francis Edward Su. "Topics in Geometric Combinatorics." Available online (http://www.cis.upenn.edu/ cis610/topics.pdf), 2004.

Edmond Jonckheere, Ming Hsieh Department of Electrical Engineering, University of Southern California, Los Angeles, CA 90089-2563, USA (jonckhee@usc.edu)

Poonsuk Lohsoonthorn, Ming Hsieh Department of Electrical Engineering, University of Southern California, Los Angeles, CA 90089-2563, USA (lohsoont@hotmail.com)

Fariba Ariaei, Department of Medicine, Division of Cardiology, Johns Hopkins University, Baltimore, MD 21287 (fariaei1@jhmi.edu)

Received July 18, 2010; accepted January 2, 2011. 